\title{
Phenotypic Heterogeneity and Plasticity of Isocortical and Hippocampal Astrocytes in the Human Brain
}

\author{
Alexander A. Sosunov, ${ }^{1}$ Xiaoping Wu, ${ }^{1}$ Nadejda M. Tsankova, ${ }^{2}$ Eileen Guilfoyle, ${ }^{2}$ Guy M. McKhann II, ${ }^{1}$ \\ and James E. Goldman ${ }^{2}$ \\ Departments of ${ }^{1}$ Neurosurgery and ${ }^{2}$ Pathology and Cell Biology, Columbia University, New York, New York 10032
}

\begin{abstract}
To examine the diversity of astrocytes in the human brain, we immunostained surgical specimens of temporal cortex and hippocampus and autopsy brains for CD44, a plasma membrane protein and extracellular matrix receptor. CD44 antibodies outline the details of astrocyte morphology to a degree not possible with glial fibrillary acidic protein (GFAP) antibodies. CD44+ astrocytes could be subdivided into two groups. First, CD44+ astrocytes with long processes were consistently found in the subpial area ("interlaminar" astrocytes), the deep isocortical layers, and the hippocampus. Many of these processes ended on blood vessels. Some were also found adjacent to large blood vessels, from which they extended long processes. We observed these $\mathrm{CD} 44+$, long-process astrocytes in every brain we examined, from fetal to adult. These astrocytes generally displayed high immunostaining for GFAP, S100 $\beta$, and CD44, but low immunostaining for glutamine synthetase, excitatory amino-acid transporter 1 (EAAT1), and EAAT2. Aquaporin 4 (AQP4) appeared distributed all over the cell bodies and processes of the CD44+ astrocytes, while, in contrast, AQP4 localized to perivascular end feet in the CD44protoplasmic astrocytes. Second, there were CD44+ astrocytes without long processes in the cortex. These were not present during gestation or at birth, and in adult brains varied substantially in number, shape, and immunohistochemical phenotype. Many of these displayed a "mixed" morphological and immunocytochemical phenotype between protoplasmic and fibrous astrocytes. We conclude that the diversity of astrocyte populations in the isocortex and archicortex in the human brain reflects both intrinsic and acquired phenotypes, the latter perhaps representing a shift from CD44- "protoplasmic" to CD44+ "fibrous"-like astrocytes.
\end{abstract}

Key words: astrocyte; CD44; interlaminar astrocyte; protoplasmic astrocyte

\section{Introduction}

Astrocytes have received increasing attention in studies of CNS structure and pathology. Much of our current knowledge of astrocytes in the normal and pathological CNS is based on studies of rodent brain. A few studies have dealt with human astrocytes and have revealed peculiar anatomical features not found in the rodent CNS, such as the presence of astrocytes with long, unbranched processes in the human CNS, described by Andriezen (1893), Retzius (1893), Cajal SRy (1984), and other pioneering neurocytologists, and recently revisited (Colombo et al., 1997; Reisin and Colombo, 2002a; Colombo and Reisin, 2004; Oberheim et al., 2009). These astrocytes are readily distinguished from "protoplasmic" astrocytes, which display a bushy morphology and approximately spherical domains. Several investigators have correlated these long-process astro-

\footnotetext{
Received Aug. 27, 2013; revised Dec. 16, 2013; accepted Jan. 3, 2014.

Author contributions: A.A.S., X.W., N.M.T., E.G., G.M.M., and J.E.G. designed research; A.A.S., X.W., N.M.T., and E.G. performed research; A.A.S., X.W., N.M.T., E.G., G.M.M., and J.E.G. analyzed data; A.A.S., X.W., N.M.T., E.G., G.M.M., and J.E.G. wrote the paper.

This research was supported by National Institutes of Health Grant NS42803 and by the Tuberous Sclerosis Alliance. We thank Dr. Markel Olabarria-Larizgoita and Dr. Marisa Cotrina for advice and discussions.

The authors declare no competing financial interests.

Correspondence should be addressed to Dr. James E. Goldman, Department of Pathology and Cell Biology, Columbia University College of Physicians and Surgeons, 630 West 168th Street, New York, NY 10032. E-mail: jeg5@columbia.edu.

DOI:10.1523/JNEUROSCI.4037-13.2014

Copyright $\odot 2014$ the authors $\quad 0270-6474 / 14 / 342285-14 \$ 15.00 / 0$
}

cytes with the presence of CD44, a receptor for extracellular matrix molecules (Bignami and Dahl, 1986; Girgrah et al., 1991; Vogel et al., 1992; Akiyama et al., 1993; da Cruz et al., 1993; Kaaijk et al., 1997; Arai et al., 2000). CD44 is also found in the "fibrous" astrocytes of white matter.

Reactive gliosis also results in increased expression of CD44, with more CD44+ astrocytes appearing in a variety of neurological disorders, such as Alzheimer's disease (Akiyama et al., 1993), argyrophilic grain disease (Botez et al., 1999), multiple sclerosis (Girgrah et al., 1991), tuberous sclerosis (Arai et al., 2000), and gliotic tissue adjacent to meningiomas (Zeltner et al., 2007). Many of these "reactive" astrocytes reside in the cortex and other gray matter areas, which are normally populated by $\mathrm{CD} 44-$, protoplasmic astrocytes. Thus, such examples of reactive gliosis might suggest that some of the CD44+ reactive astrocytes arise from CD44- protoplasmic astrocytes. Indeed, we have observed this transition in a mouse model of Alexander disease, a disorder caused by mutations in GFAP (Sosunov et al., 2013).

We characterized the immunohistochemical phenotypes of $\mathrm{CD} 44+$ astrocytes in human brain specimens (isocortex and hippocampus) obtained at surgery and at autopsy. As anticipated, we found CD44+ astrocytes with long processes at the pial surface, in deep cortical layers, and next to large cortical blood vessels. These astrocytes displayed immunocytochemical phenotypes similar to those of fibrous astrocytes of white matter and different from protoplasmic astrocytes. In addition, we found other 
CD44+ cortical astrocytes, which varied in number from specimen to specimen and displayed "mixed" morphological and immunocytochemical phenotypes between protoplasmic and fibrous astrocytes. Many of these did not have long processes. To characterize the normal development of these CD44+ populations, we examined fetal and neonatal brains. CD44+ astrocytes with pial-based long processes appeared after 26 weeks of gestation. By full term, the human brain contained $\mathrm{CD} 44+$ long-process astrocytes in white matter, deep cortex, at the pial surface, and around large vessels. However, we found no short-process CD44+ astrocytes in the cortex. Thus, the CD44+ long-process astrocytes appear intrinsic to the human brain, but the highly variable numbers of short-process CD44+ astrocytes in the adult cortex appear to be an acquired population.

\section{Materials and Methods}

Human tissue specimens. We examined 58 samples of neocortex (4 frontal, 2 parietal, and 52 temporal lobes; without radiologically and neuropathologically identified focal lesions), 11 samples of nonsclerotic hippocampi, and 14 samples of sclerotic hippocampi (mean age at surgery, 36 years; age range, 2-69 years; of either sex) obtained from surgical resections in patients with medically intractable epilepsy. Six samples were obtained from patients without seizures who had surgical resection due to cavernous malformation (two patients), aneurysm, brain abscess, epidermoid cyst, or meningioma. The studied samples did not include parenchyma with focal lesions and were considered at neuropathological examination to be normal. The mean age of patients at surgery was 66 years (age range, $34-75$ years).

Autopsy specimens of frontal and temporal cortex with underlying white matter and basal ganglia were collected from 19 to 21 weeks gestation (three specimens), at 26 weeks (two specimens), and from 38 to 40 weeks (three specimens); and at postnatal ages 3 weeks (one specimen), 7 weeks (one specimen), 6 months (one specimen), 1 year (one specimen), 2 years (two specimens), 5 years (one specimen), and 7 years (one specimen), of either sex. Samples of neocortex (temporal and parietal lobes) and hippocampi were taken at autopsy from six subjects (mean age, $47.4 \pm 4.5$ years; age range, 42-54 years; of either sex) without brain pathology. None of these brains showed evidence of neuropathology. All patient protocols were approved by the Institutional Review Board of Columbia University Medical Center.

Histology and immunohistochemistry. Surgical specimens were fixed in $4 \%$ paraformaldehyde in PBS for $12-18 \mathrm{~h}\left(4^{\circ} \mathrm{C}\right)$. The $40 \mu \mathrm{m}$ sections were prepared with a vibratome (VT1000S, Leica) and stored in cryoprotectant solution at $-20^{\circ} \mathrm{C}$. The standard procedure for Nissl staining with cresyl violet was used for routine analysis of tissue. Some autopsy tissue (fetal and

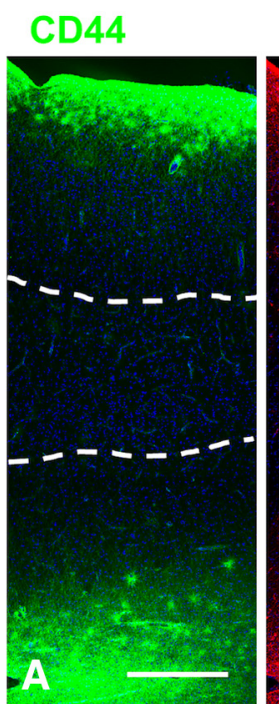

GFAP

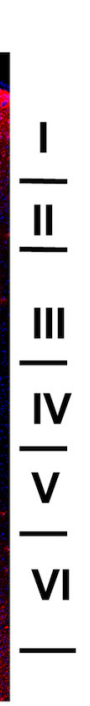

CD44
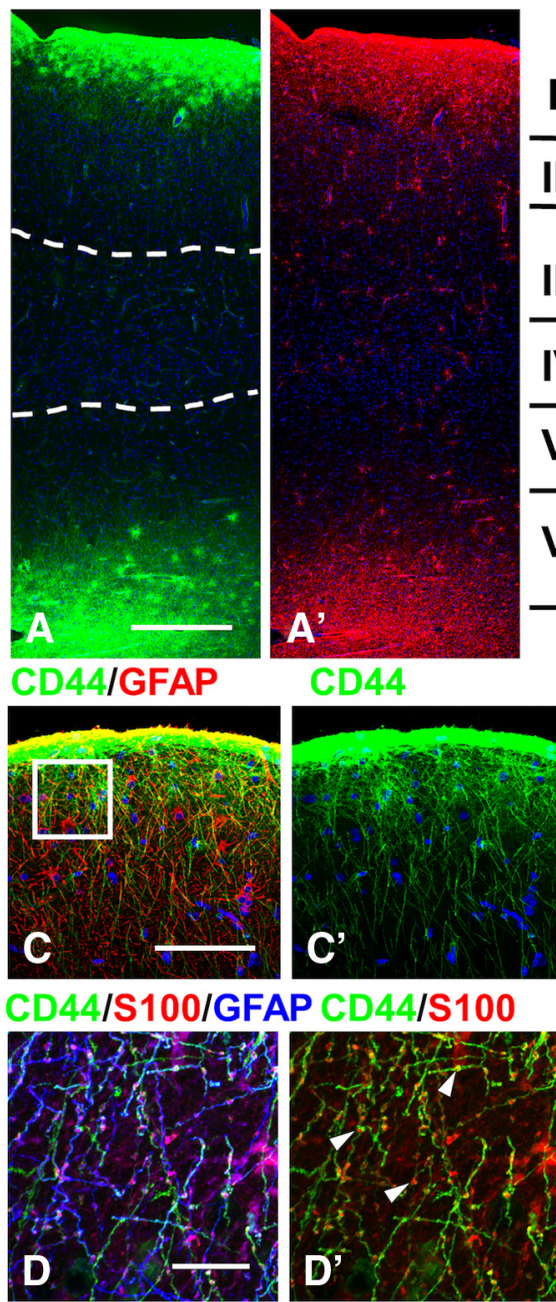

P CD44/S100
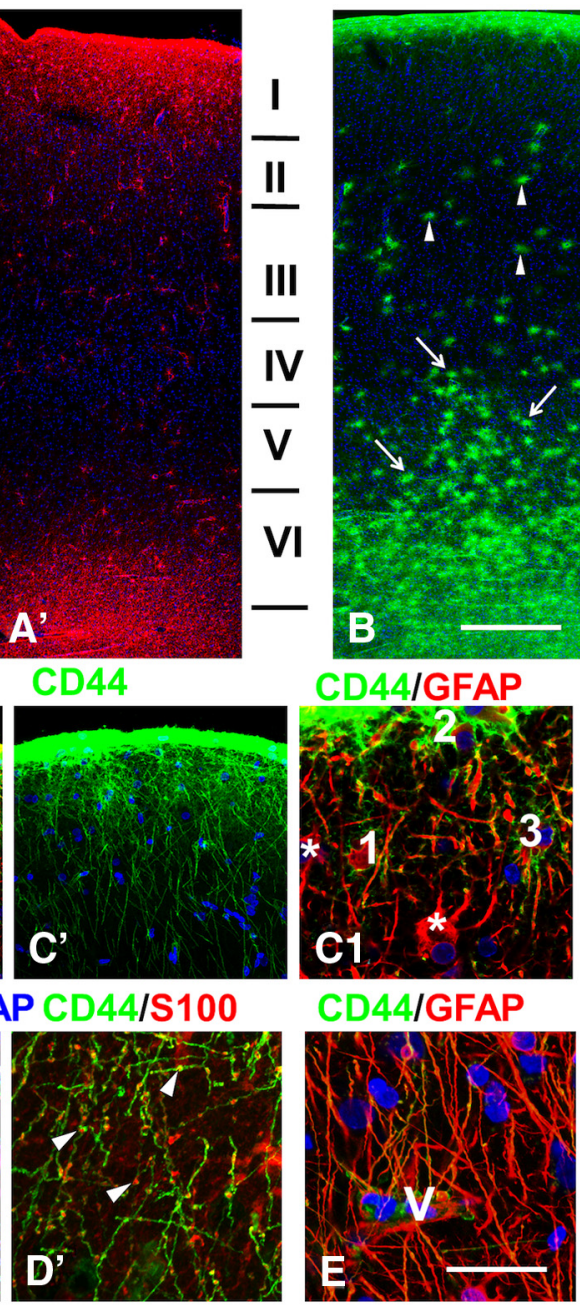

CD44/GFAP

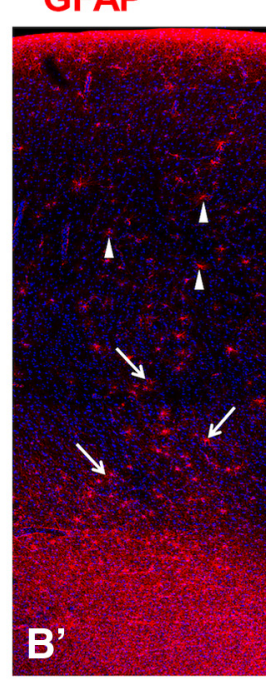

CD44

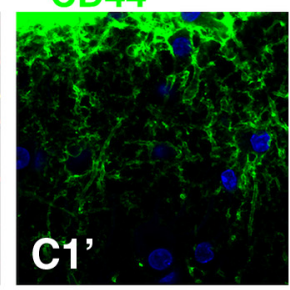

CD44
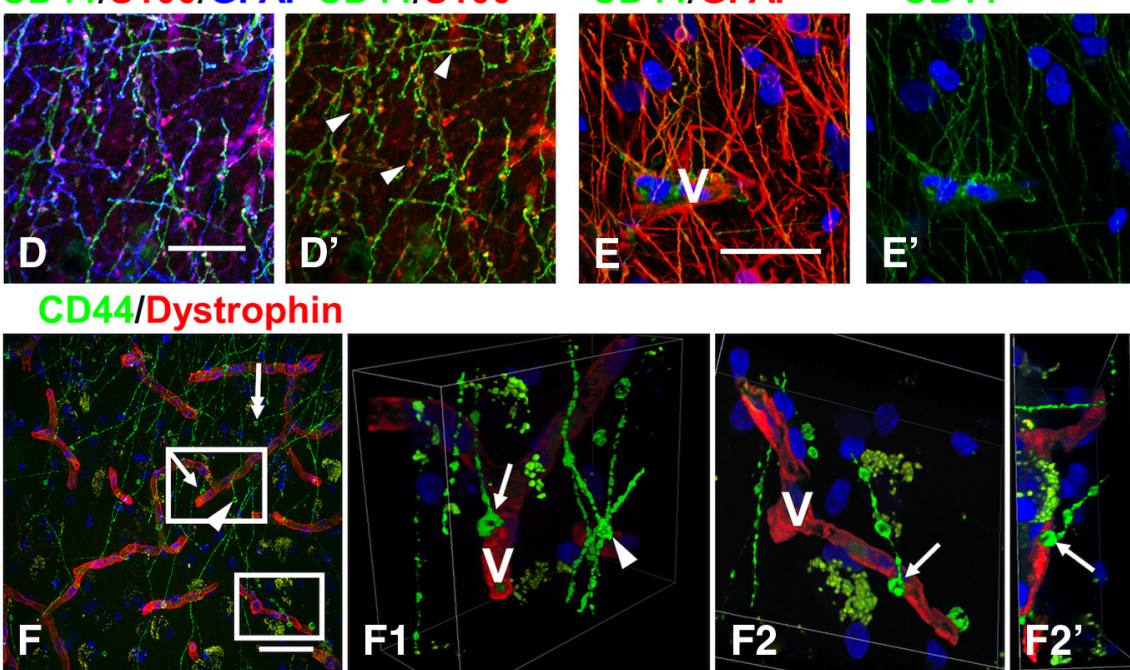

Figure 1. $\quad(D 44+$ astrocytes in human brain. $A, B, 0$ verview of two different specimens of temporal isocortex with few $(\boldsymbol{A})$ or many $(\boldsymbol{B})$ CD44+ astrocytes in the cortex. $A$, many long $C D 44+$ astrocyte processes course through upper and lower cortical layers (dotted lines define their tips in mid-cortex). Note that their lengths in upper and lower cortex are similar. I-VI in $\boldsymbol{A}^{\prime}$ indicate cortical layers. $\boldsymbol{B}$, Longprocess (arrows) and short-process (arrowheads) CD44 + astrocytes occupy most cortical layers. Only some cells were indicated. C, Subpial interlaminar astrocytes. C1, Enlarged boxed area in C; single optical slices. Note (1) that CD44+ astrocytes (1-3) have only a few main branches with clearly outlined profiles, and (2) neighboring GFAP + astrocytes (asterisks) are CD44 negative. D, Dense packing of long processes of subpial interlaminar astrocytes in layer II. Note that the majority of varicosities (arrowheads, marking only some varicosities) are immunopositive for GFAP and S100 $\beta$. E, Many long processes of subpial CD44 + astrocytes end on a blood vessel (V) in cortical layer II. $F$, Endings of long-process CD44+ astrocytes in the lower part of layer III. One CD44 + process ends with an enlargement in the neuropil (two-headed arrow). $F$ 1, 2D views of a 3D reconstruction of enlarged upper boxed area in $\boldsymbol{F}$ showing end foot (arrow in $\boldsymbol{F}$ and $\boldsymbol{F}$ ) at a vessel wall (V). Note that the varicose-like enlargement that looks like a "free ending" (arrowhead) leaves the volume of the optical imagez-stack. $F 2, F 2^{\prime}, 2 D$ views shown at different angles of a $3 D$ reconstruction of enlarged lower boxed area in $F$. V, Blood vessel visualized with dystrophin. Confocal microscopy. Scale bars: $\boldsymbol{A}, \boldsymbol{B}, 450 \mu \mathrm{m} ; \boldsymbol{C}, 115 \mu \mathrm{m} ; \boldsymbol{D}, \boldsymbol{E}, 35 \mu \mathrm{m} ; \boldsymbol{F}, 50 \mu \mathrm{m}$.

children) was fixed with $10 \%$ formalin and embedded into paraffin. Five micrometer sections were used for immunostaining. Other autopsy tissues (adult) were fixed in paraformaldehyde and sectioned at $40 \mu \mathrm{m}$ on a vibratome. 

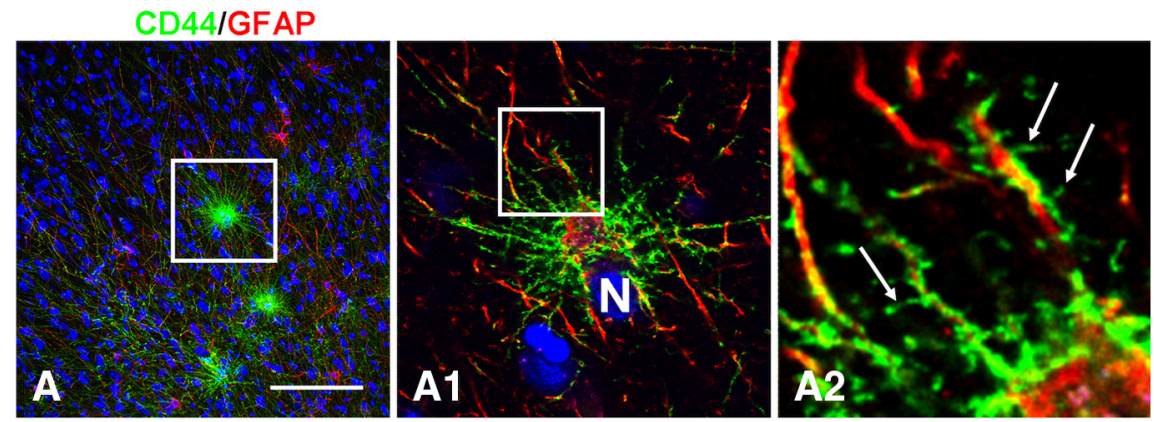
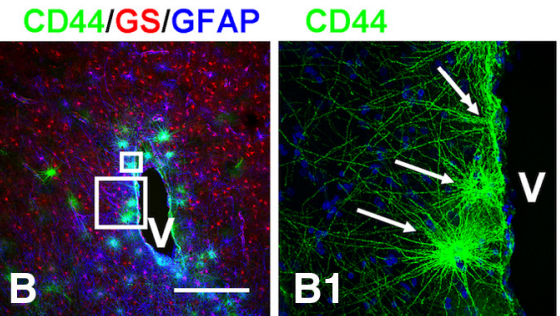

GS

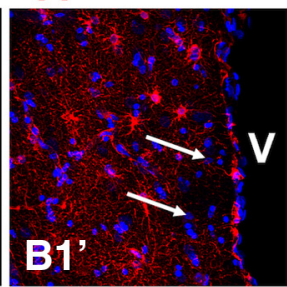

CD44/GFAP

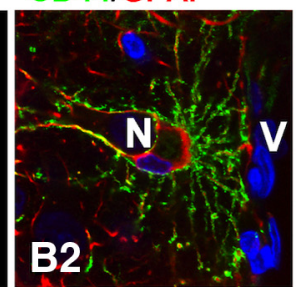

CD44/GS

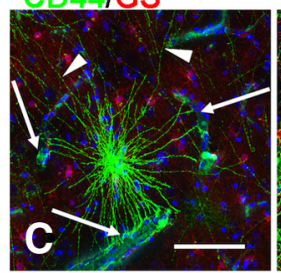

CD44/GFAP
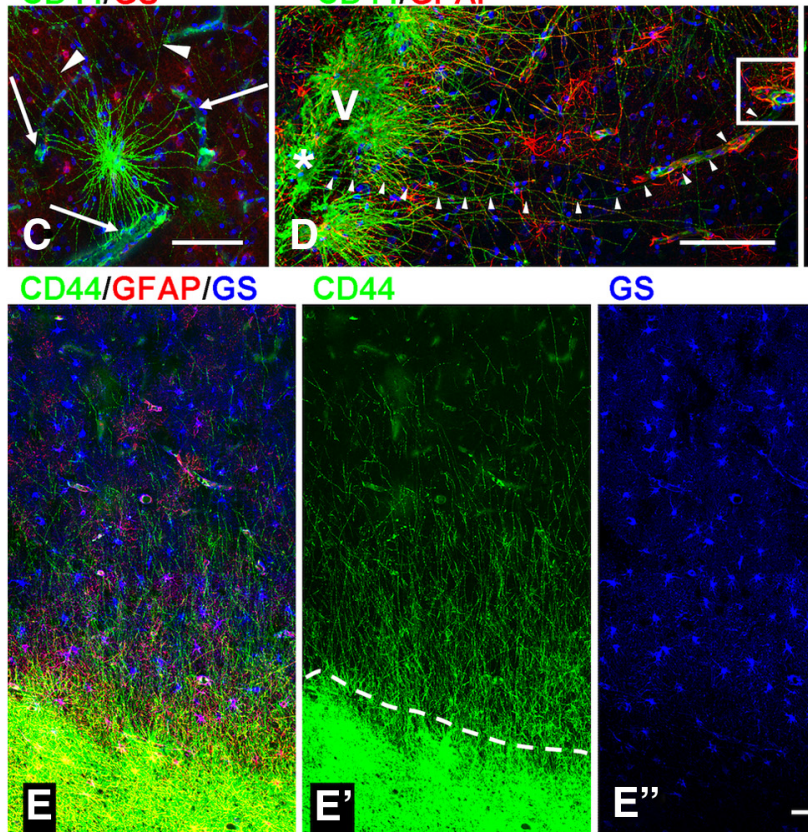

CD44
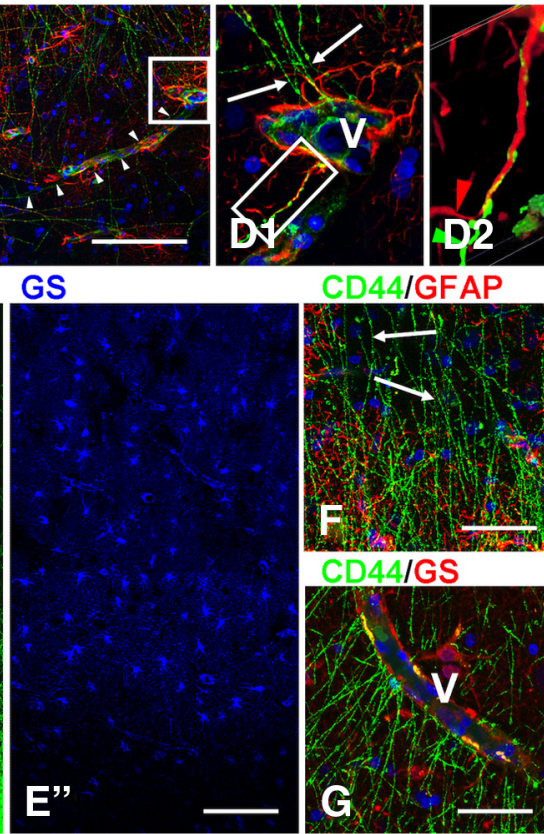

CD44/GFAP

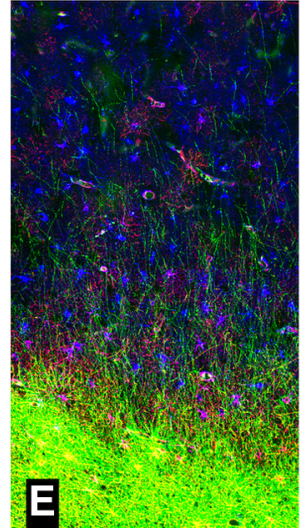

E

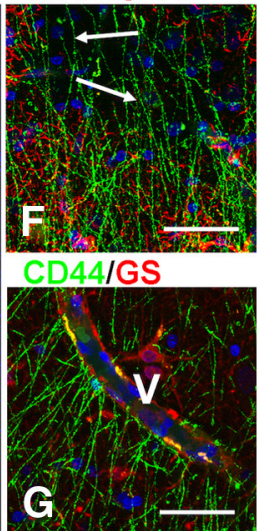

Figure 2. CD44+ astrocytes with long processes in temporal isocortex. $A$, Long-process CD44+ astrocytes in the deep cortical layers. $\boldsymbol{A} \mathbf{1}$, Enlarged boxed area in $\boldsymbol{A}$; single optical slice. Note perineuronal (N) position of CD44+ astrocyte. $\boldsymbol{A 2}$, Enlarged boxed area in $\boldsymbol{A 1}$. The main branches have only small spine-like protrusions (arrows) delineated with CD44. B, CD44+ astrocytes with long processes near a large blood vessel (V) in cortical layer IV. B1, Enlarged lower boxed area in $\boldsymbol{B}$. Pedivascular astrocytes (arrows) in $\boldsymbol{B} \mathbf{1}^{\prime}$ have low levels of GS; double-headed arrow indicates perivascular astrocyte with long processes. $\boldsymbol{B 2}$, Enlarged upper boxed area. The astrocyte that occupies a perineuronal (N) position sends several end feet to blood vessel (V). C, Astrocyte in layer IV sends end feet to several blood vessels (arrows), whereas other processes pass over these vessels (arrowheads) and end beyond the area of the image. $\boldsymbol{D}$, A long process (marked with arrowheads) of a perivascular (V) astrocyte (asterisk) ends on small blood vessels (see in D1, enlarged boxed area in D), Note in D1 that several CD44 + processes (arrows) going from upper parts of cortex make end feet on the same vessel (V). D2, Enlarged boxed area in D1 shows that the long CD44+ process "crawls" along a CD44-/GFAP+ astrocyte process approaching the vessel. CD44+ and GFAP + processes are marked with green and red arrowheads, respectively, before they touch each other. $\boldsymbol{E}$, Long processes ascending from white matter astrocytes into lower cortical layers. The gray matter-white matter border is defined with a dotted line $\left(\boldsymbol{E}^{\prime}\right)$ and is clearly discriminated by CD44 and GS immunostaining. $\boldsymbol{F}$, Many thin astrocyte processes in lower cortical layers do not show detectable levels of GFAP immunostaining (arrows, marking only some processes). $G$, Many long astrocyte processes in the lower cortical layers end on blood vessels (V). Confocal microscopy. Scale bars: $\boldsymbol{A}, 180 \mu \mathrm{m} ; \boldsymbol{B}, 215 \mu \mathrm{m} ; \boldsymbol{C}, \boldsymbol{D}, 120 \mu \mathrm{m} ; \boldsymbol{E}, 85 \mu \mathrm{m} ; \boldsymbol{F}, 40 \mu \mathrm{m} ; \boldsymbol{G}, 30 \mu \mathrm{m}$.

Antibodies. Primary antibodies were used against the following: (1) CD44, mouse monoclonal (1:80; clone F10-44-2, Dako) and rat monoclonal (1:100; clone A020, Millipore); (2) glial fibrillary acidic protein (GFAP), mouse monoclonal (1:1000; G3893, Sigma-Aldrich), rabbit polyclonal (1:1000; Z 0334, Dako), and chicken polyclonal (1:500; PCK-591P, Covance); (3) vimentin, mouse monoclonal (1:500; M 0725, Dako); (4) nestin, rabbit polyclonal (1:500; PRB-570, Covance); (5) glutamine synthetase (GS), mouse monoclonal (1:1000; MAB302, Millipore) and rabbit polyclonal (1:200; sc9067, Santa Cruz Biotechnology); (6) astrocytespecific glutamate transporters, excitatory amino-acid transporter 1 (EAAT1; GLAST), monoclonal (1:100; clone 10D4, Novocastra Laboratories); EAAT2 (GLT-1), mouse monoclonal (1:500; 611654, BD Transduction Laboratories); (7) aquaporin 4 (AQP4), rabbit polyclonal (1:200; 178614, Calbiochem); (8) calcium-binding protein specific for glial cells (S100), rabbit polyclonal (1:600; A 5110, Dako); (9) $\alpha$ B-crystallin, rabbit polyclonal (1: 300; SPA-223, Stressgen); (10) phospho-S6 ribosomal protein (Ser235/236), rabbit polyclonal (1:100; 4857, Cell Signaling Technology); (11) synaptophysin, rabbit polyclonal (1: 250; A 0010, Dako); (12) dystrophin, mouse monoclonal (1:300; D8043, Sigma); (13) myelin basic protein, rabbit polyclonal (1:200; A0623, Dako); (14) Caspr1, sheep polyclonal (1:100; AF7548, R\&D Systems); and (15) Lucifer yellow, rabbit polyclonal (1:200; AB154, Millipore). Secondary antibodies used were conjugated to fluorophores and included anti-chicken Alexa Fluor 594 and 633; antimouse Alexa Fluor 488, 594, and 633; antirabbit Alexa Fluor 594; and anti-rat Alexa Fluor 488 and 594, all from goat or donkey (1:300; Invitrogen).

For light microscopy, sections were pretreated in $3 \% \mathrm{H}_{2} \mathrm{O}_{2}$ in methanol (30 min) and after blocking in $10 \%$ donkey serum incubated overnight $\left(4^{\circ} \mathrm{C}\right)$ with primary antibodies. After washing, sections were incubated with biotinylated secondary antibodies (1:1000; Jackson ImmunoResearch) for $1 \mathrm{~h}$ at room temperature (RT) and then, after washing, with avidin-biotin-peroxidase complex (Vector Laboratories), for $1 \mathrm{~h}$ at RT. Peroxidase activity was visualized with $0.03 \%$ DAB (Sigma) with $0.005 \% \mathrm{H}_{2} \mathrm{O}_{2}$ in $0.05 \mathrm{~m}$ Tris buffer. Sections were mounted on glass slides, dehydrated in a graded ethanol series, and mounted in DPX medium. For double/triple immunofluorescence, after blocking with $10 \%$ normal goat (or donkey) serum (for $30 \mathrm{~min}$, at RT), freefloating sections were incubated in a mixture of primary antibodies raised in different species overnight $\left(4^{\circ} \mathrm{C}\right)$. For visualization, Alexa Fluor-conjugated secondary antibodies were used for $1 \mathrm{~h}$ at RT. Fluorescent Nissl reagent (1:150; NeuroTrace 640/660 deep-red, Invitrogen) was used (for $30 \mathrm{~min}$, at RT) for visualization of general histological structures in double immunostaining. DAPI (Vector Laboratories) was used with triple immunostaining. Blocking serum, primary, secondary antibodies, and fluorescent Nissl reagent were applied in $0.2 \%$ Triton X-100 in PBS. Sections for fluorescent microscopy were mounted on slides in Vectashield (Vector Laboratories). To control the specificity of immunostaining, primary antibodies were omitted and substituted with appropriate normal serum. Slides were viewed using a Nikon A1R MP 
A control astrocyte astrocyte with long processes

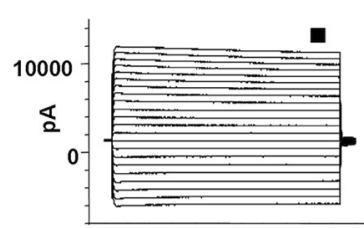

$50 \mathrm{~ms} 100$
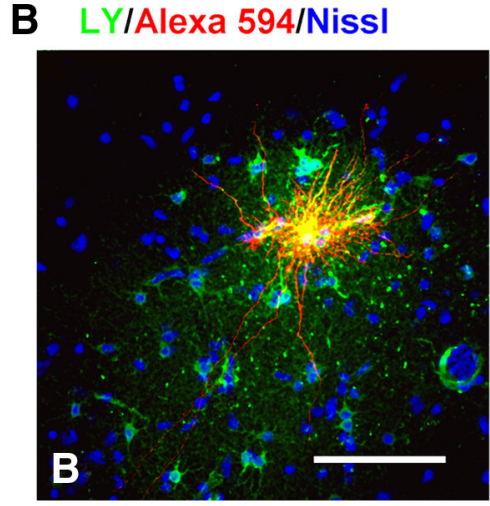

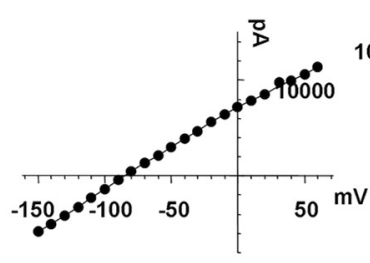

Alexa 594

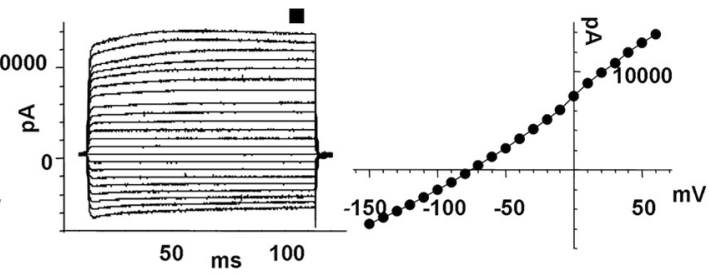

Alexa 594
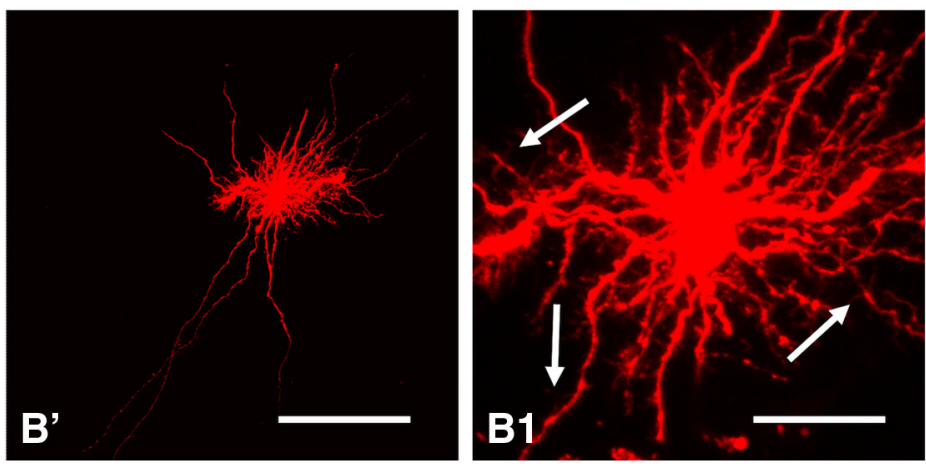

Figure 3. A, Representative current traces were evoked by a step voltage protocol (from -160 to $+60 \mathrm{mV}$ in $10 \mathrm{mV}$ increments; $100 \mathrm{~ms}$ duration). The current-voltage ( $I-V$ ) curves were obtained from the measurements near the end of each voltage step (small black squares above current traces). Note the absence of difference between control (short processed astrocyte, left) and astrocyte with long processes (right). $\boldsymbol{B}$, A subpial interlaminar astrocyte was filled with Lucifer yellow (for intercellular coupling) and with Alexa Fluor 594 (which does not penetrate gap junctions). Many neighboring astrocytes were filled with Lucifer yellow $(\boldsymbol{B})$. Cell processes of the injected astrocyte have short spine-like protrusions (arrows in $\boldsymbol{B} 1$, enlargement of $\boldsymbol{B}^{\prime}$ ). Confocal microscopy. Scale bars: $\boldsymbol{B}, \boldsymbol{B}^{\prime}, 125 \mu \mathrm{m} ; \boldsymbol{B} \mathbf{1}, 25 \mu \mathrm{m}$.

confocal microscope. 3D reconstructions were generated from stacks of images with the confocal microscope software NIS-Elements.

Quantitative immunohistochemical analysis. The numbers of CD44immunolabeled cells were quantified in the images merged from stacks of adjacent six images $(1024 \times 1024$ pixel resolution; observed area, $606 \times$ $606 \mu \mathrm{m}$ ) captured at a distance of $0.5 \mu \mathrm{m}$ from each other. Only cells with clearly outlined nuclei were taken into consideration. Three randomly selected areas from mid-cortical layers (layers III and IV; for analysis of CD44+ astrocytes without long processes) and deep layers (layers $\mathrm{V}$ and VI; for analysis of CD44+ astrocytes with long processes) from 32 specimens were used. These specimens were selected because they contained many CD44+ astrocytes in mid-cortical layers.

To quantitate the long CD44+ processes of interlaminar astrocytes that end either on blood vessels or are "free" within the neuropil, $z$-stacks of optical slices were taken from the entire depth of the $40 \mu \mathrm{m}$ sections with optical sections of $0.5 \mu \mathrm{m}$ (except areas near the surface to avoid nonspecific staining) from the $295 \times 295 \mu \mathrm{m}$ area with $1024 \times 1024$ pixel resolution. To identify blood vessels, we used immunostaining for dystrophin, which defined most if not all vessels. The positions of CD44+ astrocyte processes were determined in 3D reconstruction of the areas of interest. Only those processes whose terminal parts (usually varicose-like enlargements) did not contact the marginal surfaces of the examined volume or blood vessels were considered as free endings.

To assess the GS immunostaining levels in CD44+ astrocytes with long processes versus CD44- protoplasmic astrocytes, images (merged three adjacent optical slices captured at a distance of $0.25 \mu \mathrm{m}$ from each other, with $1024 \times 1024$ pixel resolution, from a $295 \times 295 \mu \mathrm{m}$ area) were obtained from double immunostained GS and CD44 slices. Images were transferred to ImageJ 1.47 (public domain), and the optical density (OD) was determined in a circular area with diameter of $100 \mu \mathrm{m}$ [this size was selected to minimize inclusion of neighboring astrocytes processes, given that a diameter of a human protoplasmic astrocyte domain is $\sim 140 \mu \mathrm{m}$ (Oberheim et al., 2009)].

For correlation between CD44 and GFAP immunostaining images (stacks of adjacent six optical slices captured at a distance of $0.5 \mu \mathrm{m}$; $1024 \times 1024$ pixel resolution; observed area, $606 \times 606 \mu \mathrm{m}$,) were obtained from the whole depth of the neocortex. Three randomly selected areas from each of 32 specimens were used for analysis of OD with ImageJ. Correlation between levels of CD44 and GS immunostaining in $\mathrm{CD} 44+$ astrocytes without long processes was performed on images obtained from stacks of three adjacent optical slices captured at a distance of $0.25 \mu \mathrm{m}$, with $1024 \times 1024$ pixel resolution, and an observed area of $295 \times 295 \mu \mathrm{m}$. It should be noted that these areas included many CD44- protoplasmic astrocytes. The OD was determined with ImageJ. Quantification of synaptophysin-positive puncta was performed on the images obtained from stacks of three adjacent optical slices captured at a distance of $0.25 \mu \mathrm{m}$, with $1024 \times 1024$ pixel resolution, and an observed area of $98 \times 98 \mu \mathrm{m}$. Areas of interest $(30 \times 30 \mu \mathrm{m})$ were selected near cell bodies (excluding perikarya) of $\mathrm{CD} 44+$ astrocytes and neighboring CD44- protoplasmic astrocytes, and were used for analysis of the OD with ImageJ.

Electrophysiological recording in acute brain slices. Freshly obtained samples of surgically resected tissue were cut with a vibratome (VT 1000 S, Leica) in ice-cold oxygenated-modified artificial CSF containing the following (in $\mathrm{mm}$ ): $125 \mathrm{NaCl}, 2.5 \mathrm{KCl}, 2 \mathrm{CaCl} 2,1.5 \mathrm{MgCl} 2,1.25$ $\mathrm{NaH}_{2} \mathrm{PO}_{4}, 26 \mathrm{NaHCO} 3$, and 10 dextrose. The slices $(160-180 \mu \mathrm{m})$ were recovered at $30^{\circ} \mathrm{C}$ in a chamber for at least $1 \mathrm{~h}$ before electrophysiological recording. The above solution was also used for whole-cell patchclamp recording in brain slices at $30^{\circ} \mathrm{C}$. The bath solution was applied at a flow rate of $1.5 \mathrm{ml} / \mathrm{min}$ using the VC-6 perfusion valve control system (Warner Instruments) with the TC-344B temperature controller. Cells were visualized under a Leica DMLFS microscope with a $63 \times$ waterimmersion lens. The intracellular solution in the patch pipettes contained the following (in mM): $140 \mathrm{KCl}, 1 \mathrm{MgCl} 2,10 \mathrm{EGTA}, 10 \mathrm{HEPES}, 3$ MgATP, and 0.3 Na2ATP, pH 7.3 with $\mathrm{KOH}$. The $1 \mu \mathrm{M}$ tetrodotoxin (Sigma) was applied in the bath solution to block sodium channels. Pipette resistance was $\sim 3-5 \mathrm{M} \Omega$. Cell capacitance and series resistance were measured using the software MultiClamp 700A Commander, version 1.1.2.27, and pClamp 8 (Axon Instruments). Cells were initially identified morphologically based on the sizes and shapes of their somas, and the architecture of their processes. For analysis of cell morphology and gap junction coupling with the surrounding cells, Lucifer yellow (Sigma-Aldrich) and Alexa Fluor 594, which does not penetrate through gap junctions and thus allow us to evaluate the shape of the patched cell, 


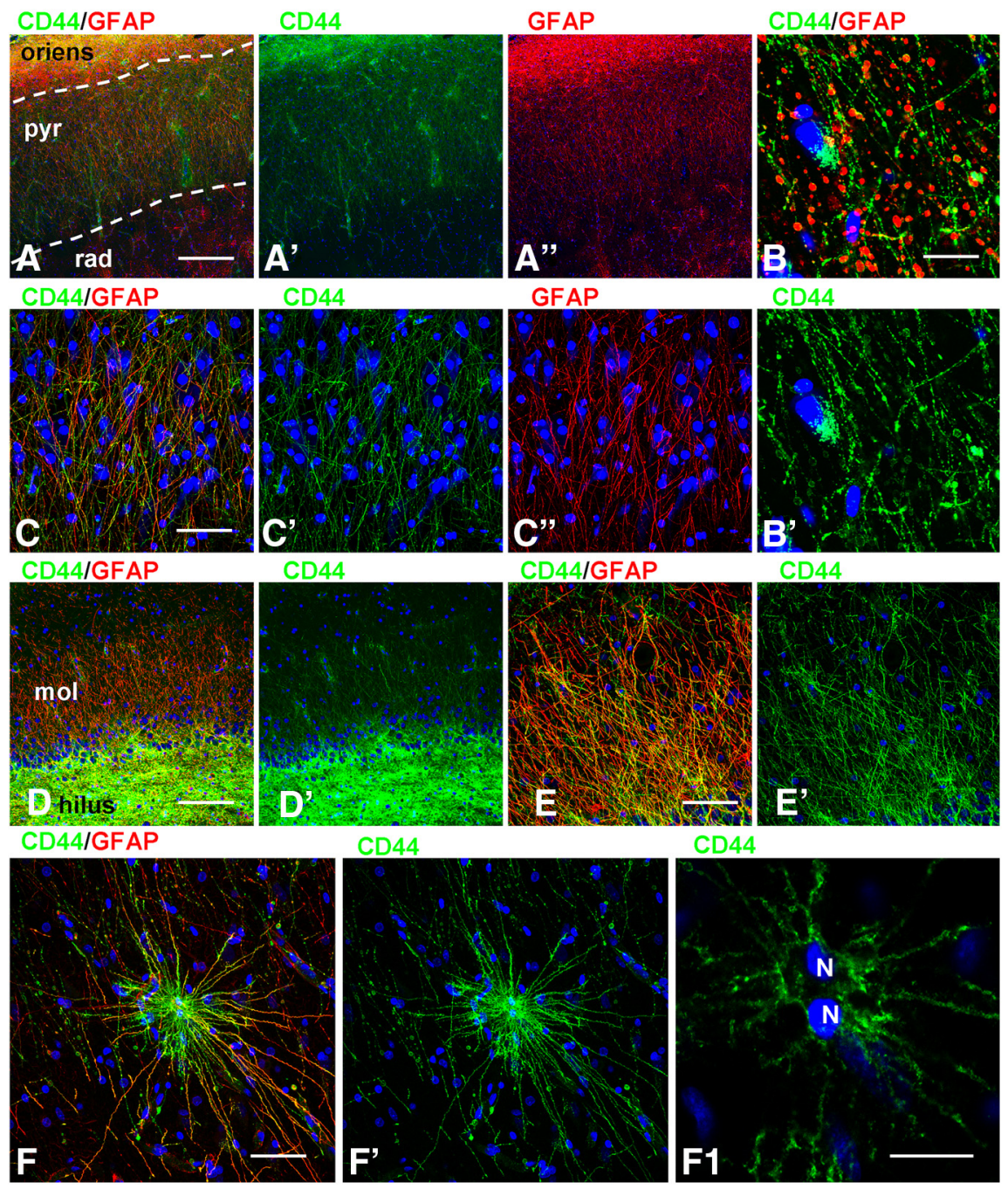

Figure 4. CD44+ astrocytes in hippocampus. $\boldsymbol{A}, \boldsymbol{C}$, A dense net of long astrocyte processes covers the pyramidal layer in CA1. $\boldsymbol{A}$, Dotted lines outline stratum pyramidale (pyr): surgical tissue. $\boldsymbol{B}$, Many large varicosities in long astrocyte processes in stratum pyramidale: autopsy tissue. $\boldsymbol{D}, \boldsymbol{E}, \mathbf{C D} 44+$ processes cover the molecular layer of the dentate gyrus (mol). $\boldsymbol{F}, \mathrm{A}$ CD44+ astrocyte with long processes in the CA1 stratum radiatum. Note that this astrocyte has two nuclei (N) $\left(\boldsymbol{F} 1\right.$, enlargement of $\boldsymbol{F}^{\prime}$; single optical slice). oriens, Stratum oriens; rad, stratum radiale. Confocal microscopy. Scale bars: $\boldsymbol{A}, 210 \mu \mathrm{m} ; \boldsymbol{B}, 25 \mu \mathrm{m} ; \boldsymbol{C}, \boldsymbol{D}, 120 \mu \mathrm{m} ; \boldsymbol{E}, 35 \mu \mathrm{m}$; $\boldsymbol{F}, 30 \mu \mathrm{m} ; \boldsymbol{F 1}, 15 \mu \mathrm{m}$.

were added to the intracellular solution (final concentrations, $0.1 \%$ and $0.1 \%$, respectively) and filtered through a $0.2 \mu \mathrm{m}$ polytetrafluoroethylene filter. After the experiment, slices were immersed in fixative (4\% paraformaldehyde in PBS) and kept overnight at $4^{\circ} \mathrm{C}$. Slices were immunostained and observed under a confocal microscope, as cited above.

Statistical analysis. Data were expressed as the mean \pm SEM. Student's $t$ test and Pearson correlation coefficient were used as appropriate. $p<$ 0.05 was considered to be significant.

\section{Results}

The adult human neocortex contains CD44+ astrocytes with long processes

In every sample of neocortex, we observed CD44+ astrocytes with long processes. CD44+ astrocytes located at the pial surface and those in the lower cortical layers and superficial white matter displayed long, unbranched processes oriented orthogonally to the pial surface and to the gray matter-white matter border. The CD44+ astrocytes located at the pial surface produced processes that descended into the cortex, reaching as deep as layers III-IV (Fig. 1A-E). We observed many processes of these "interlaminar" astrocytes ending on blood vessels (Fig. $1 E, F$ ), although the end- ings of some processes were undetermined because they went beyond the borders of the section. The long processes of these astrocytes lacked secondary branches and in some specimens showed irregularly spaced, varicose-like enlargements (Fig. $1 D, D^{\prime}$ ). The varicosities have been described as a feature of the longprocess astrocytes (Oberheim et al., 2009). The cell bodies of most of these astrocytes were found within a short distance from the pia, and some $(20 \%, 58$ of 292 cells) were in direct apposition to the pia. Not all astrocytes near the pial surface were $\mathrm{CD} 44+$, however (Fig. $1 C 1, C 1^{\prime}$ ), but these did not produce long processes. We quantitated the numbers of CD44+ processes that ended either on blood vessels or ended free, without contacts with blood vessels. Because the density of interlaminar processes decreased as they traveled through the cortical layers, we examined the ends of processes in the upper part of layer III, where the density of the processes is high, and in the lower part of layer III, where the density is much lower. In the upper layer, most of the long CD44+ processes ended on blood vessels (49 of a total of 52 that were examined with $3 \mathrm{D}$ reconstruction, and 3 processes that ended free without contact with the vessels). In the lower part of layer III, we found 61 processes with end feet on blood vessels and 23 that ended with varicoselike enlargements free in the neuropil.

CD44+ astrocytes with long processes were also regularly observed in deep cortical layers (layers V and VI; "stellate independent cells"; Cajal SRy, 1984), "varicose projection astrocytes" (Oberheim et al., 2009), and superficial white matter (Figs. $1 A, B, 2 E, F)$. Many of these projected processes radially into the cortex, producing a meshwork of thin processes in layers V and VI (Akiyama et al., 1993). The processes of these cells lacked secondary branches and generated only a few spine-like protrusions in the proximal parts of processes (Fig. 2A2). As was the case for the descending processes, it was not always clear whether these ascending processes reached specific targets, but we observed many touching the walls of blood vessels (Fig. $2 G$ ). Note that the CD44+ long-process astrocytes typically send many thin processes to vessels and that their end feet appear to be small (Figs. 1E, 2B2,C,D1,G).

We found long-process astrocytes with cell bodies within the cortex. The majority of these were associated with large blood vessels (Fig. $1 B$, astrocytes along the descending blood vessel, $2 B, B 1)$. Some of their cell bodies touched blood vessel walls ("pedivascular" astrocytes, according to Cajal SRy, 1984), although more were located in the vicinity of blood vessels ("perivascular" astrocytes, according to Cajal SRy, 1984) and sent several vascular end feet to the nearest vessel (Fig. 2B-D). These astrocytes also sent long processes to terminate on blood vessels some distance away (Fig. $2 C, D, D 1, D 2$ ). 

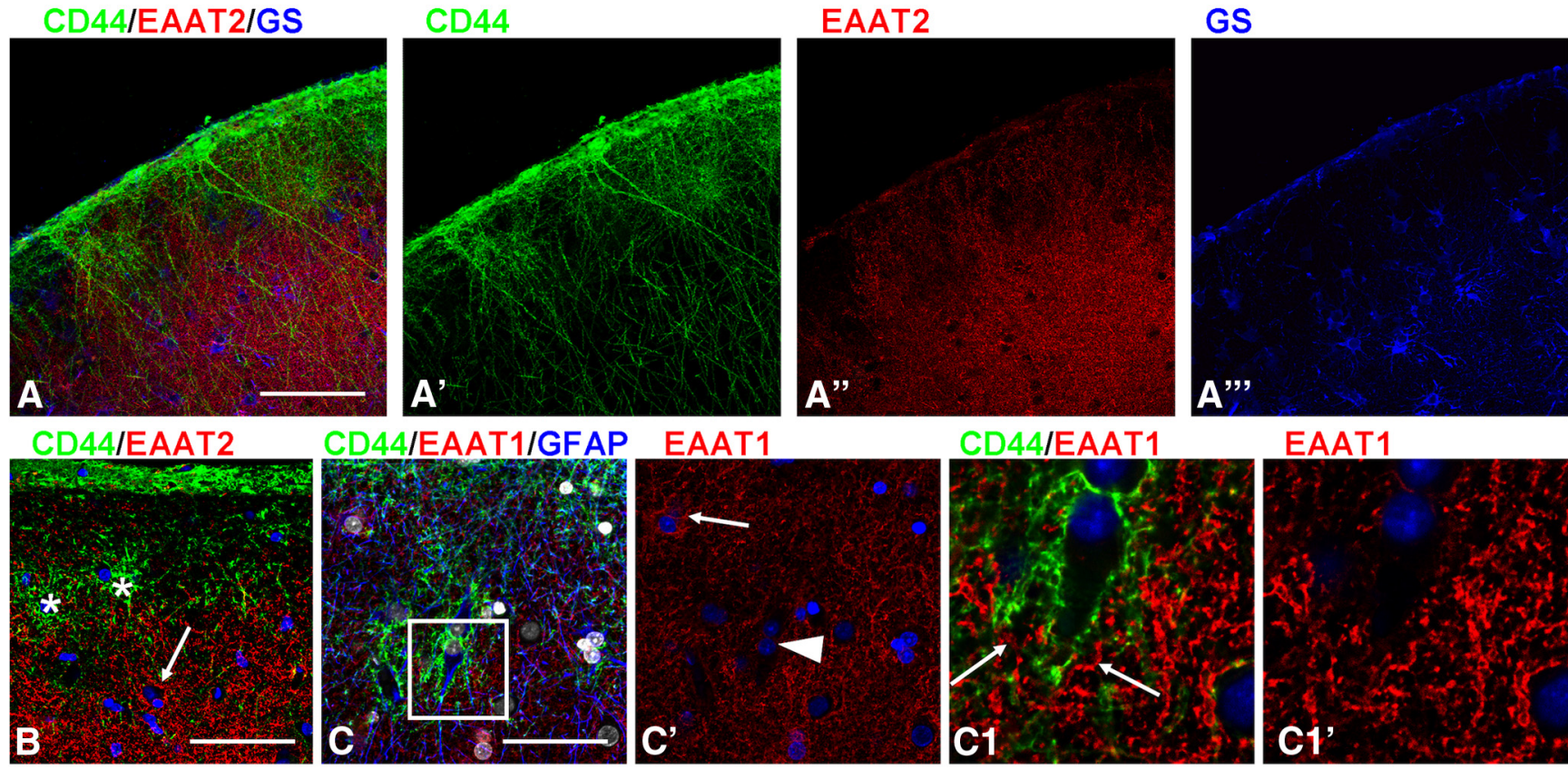

CD44/EAAT1

EAAT1

CD44/EAAT1/GFAP CD44/EAAT1
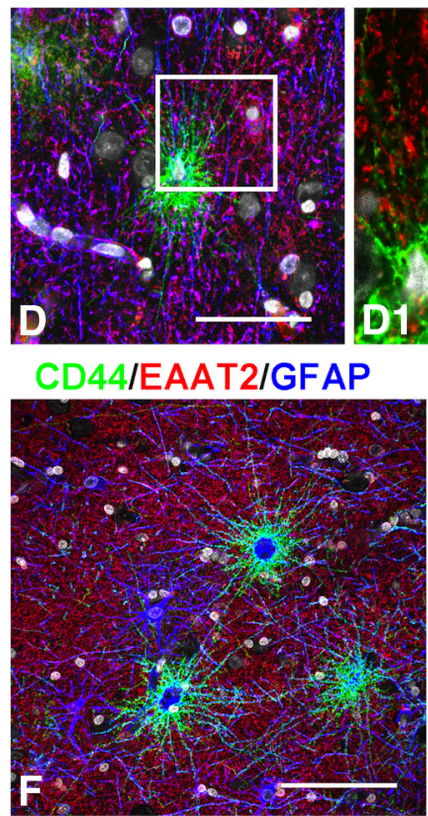

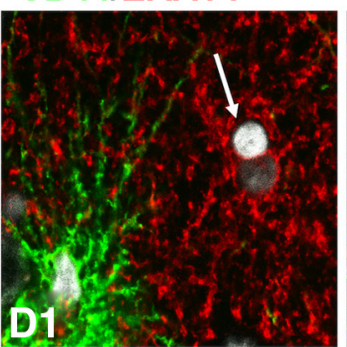

CD44.

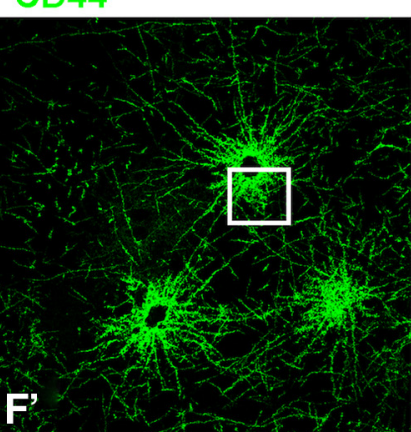

CD44/GS

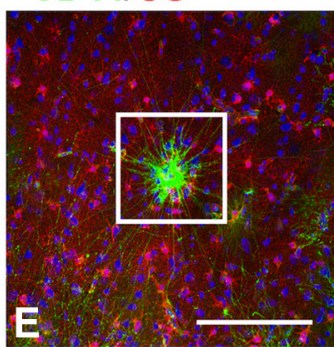

CD44/EAAT2/GFAP

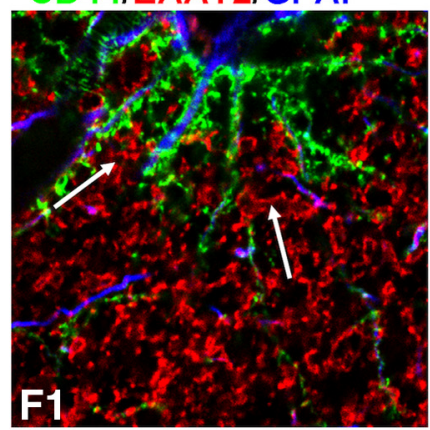

CD44/GS
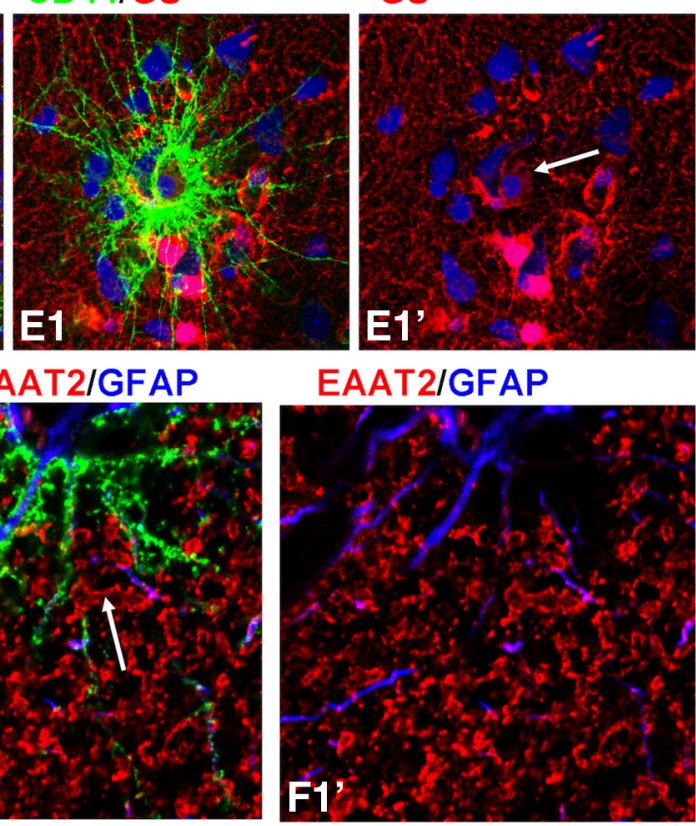

Figure 5. CD44 + astrocytes with long processes display a phenotype similar to that of fibrous astrocytes. A, EAAT2 (red) and GS (blue) are low in subpial CD44+ (green) astrocytes. $B$, Subpial CD44+ astrocytes (asterisks) display much less EAAT2 immunolabeling than neighboring CD44- astrocyte (arrow). Single optical slice. C, EAAT1 signal is low in subpial CD44+ astrocytes with long processes. Compare CD44+ astrocyte (arrowhead) and CD44- astrocyte (arrow). C, Single optical slice. C1, Enlarged boxed area in C. Blue: C, GFAP; $\boldsymbol{C}^{\prime}, \mathbf{C 1}$, $\mathbf{C 1}^{\prime}$, nuclei. Note that EAAT1 + profiles (arrows) are located in the immediate vicinity of the CD44+ astrocyte cell body. D, Low level of EAAT1 immunolabeling of a CD44+ astrocyte with long processes in cortical layer V. D1, Enlarged boxed area in $\boldsymbol{D}$, single optical slice, protoplasmic EAAT1 +/CD44 - astrocyte marked with arrow. $\boldsymbol{E}$, $\boldsymbol{F}$, Levels of GS $(\boldsymbol{E})$ and EAAT2 $(\boldsymbol{F})$ are low in CD44+ astrocytes with long processes in deep cortical layers. $\boldsymbol{E 1}, \boldsymbol{F 1}$, Enlarged boxed areas in $\boldsymbol{E}$ and $\boldsymbol{F}^{\prime}$, respectively. $\boldsymbol{E} \mathbf{1}^{\prime}, \mathrm{CD}_{44}+$ astrocyte is marked with arrow. $\boldsymbol{F 1}$, Note that EAAT2 + processes (arrows) of an adjacent CD44 - astrocyte are located near the cell body of the CD44+ astrocyte and in between the CD44+ processes. Confocal microscopy. Scale bars: $\boldsymbol{A}, 110 \mu \mathrm{m} ; \boldsymbol{B}, 55 \mu \mathrm{m} ; \boldsymbol{C}, 35 \mu \mathrm{m} ; \boldsymbol{D}, 110 \mu \mathrm{m} ; \boldsymbol{E}, 175 \mu \mathrm{m} ; \boldsymbol{F}, 130 \mu \mathrm{m}$.

Other cell bodies of CD44+ long-process astrocytes in the cortex were found intimately associated with neuronal cell bodies (Fig. $2 A 1, B 2 ; 45 \%, 123$ of 278 examined cells). We also found highly variable numbers of CD44+ astrocytes in mid-cortex that did not have long processes. Many of these astrocytes are shown at low magnification in Figure $1 B$ (unmarked by arrows). Note that another specimen does not show these CD44+ astrocytes (Fig. 1A). These cortical CD44+ astrocytes without long processes are discussed below.
We recorded from several of the long-process astrocytes in the excised temporal lobes. All displayed passive current-voltage relationships (Fig. 3A). Furthermore, we compared the passive electrophysiological properties to those of protoplasmic astrocytes and found no significant differences in resting membrane potential, input resistance, or membrane capacitance. One half (three of six) of the long-process astrocytes near the pial surface from which we recorded were coupled to neighboring protoplasmic astrocytes (Fig. $3 B$ ). In contrast, all 


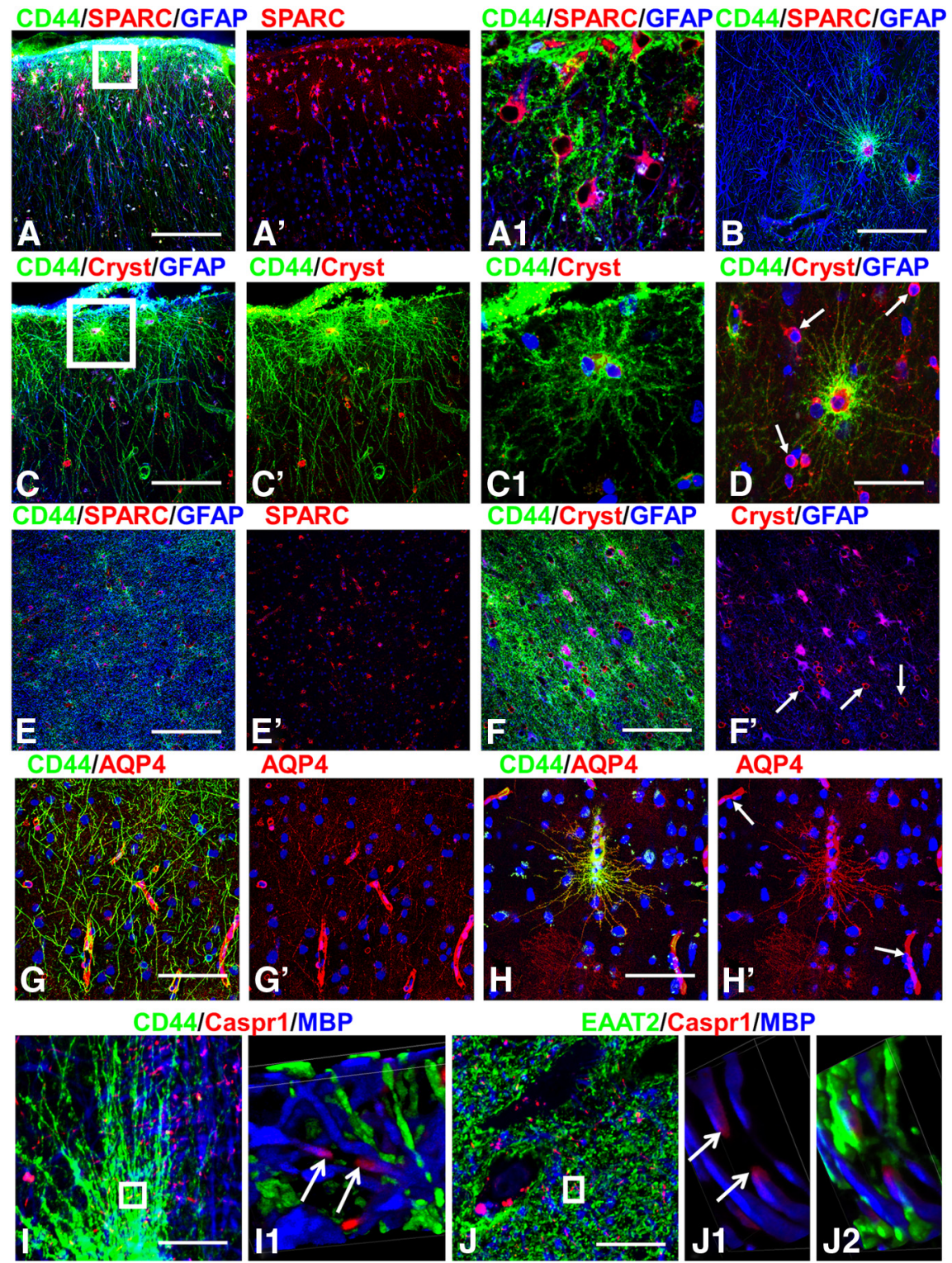

Figure 6. $\quad \boldsymbol{A}-\boldsymbol{D}, \operatorname{SPARC}(\boldsymbol{A}, \boldsymbol{B})$ and $\alpha \mathrm{B}$-crystallin (Cryst; $\boldsymbol{C}, \boldsymbol{D})$ are present in long-process astrocytes, shown in subpial $(\boldsymbol{A}, \boldsymbol{C})$ and in deep cortical layers $(\boldsymbol{B}, \boldsymbol{D})$. Cryst + oligodendrocytes in $\boldsymbol{D}$ are marked with arrows. $\boldsymbol{A} 1, \mathbf{C 1}$, Enlarged boxed areas from $\boldsymbol{A}$ and $\boldsymbol{C}$, respectively. SPARC $(\boldsymbol{E})$ and $\alpha \mathrm{B}$-crystallin $(\boldsymbol{F})$ in subcortical white matter. Arrows in $\boldsymbol{F}^{\prime}$ mark oligodendrocytes. $\boldsymbol{G}, \boldsymbol{H}, \mathrm{AQP4}$ is observed in all processes in CD44+ astrocytes. $\mathbf{G}$, Long processes of interlaminar astrocytes are AQP4 + . $\boldsymbol{H}$, Long-process CD44+ astrocyte near blood vessel displays immunoreactivity for AQP4 in processes. Note high levels of AQP4 near blood vessels (arrows). I, CD44+ long process astrocytes do not contact nodes of Ranvier visualized as the unstained part of the axon (depicted with arrows in I1) between two Caspr1+ ends of neighboring myelin sheaths. I1, $2 \mathrm{D}$ views of $3 \mathrm{D}$ reconstruction of enlarged boxed area in $\mathbf{I}$. J, Processes of protoplasmic astrocytes visualized with EAAT2 located close to a node. $\mathbf{J 1}, \mathbf{J 2}, 2 \mathrm{D}$ views of $3 \mathrm{D}$ reconstruction of enlarged boxed area in $J$. Note that EAAT2 + (green)-immunopositive processes are near the node (defined as space between arrows). Confocal microscopy. Scale bars: $\boldsymbol{A}, \boldsymbol{B}, 130 \mu \mathrm{m} ; \boldsymbol{C}, 100 \mu \mathrm{m} ; \boldsymbol{D}, 55 \mu \mathrm{m} ; \boldsymbol{E}, 130 \mu \mathrm{m} ; \boldsymbol{F}, 130 \mu \mathrm{m} ; \boldsymbol{G}, 85 \mu \mathrm{m}$; H, $120 \mu \mathrm{m}$.

protoplasmic astrocytes were coupled to neighboring protoplasmic astrocytes.

\section{The human hippocampus also contains CD44+ astrocytes} with long processes

We found CD44+ astrocytes in the stratum oriens of the hippocampus that projected long processes covering the entire depth of the pyramidal layer of CA1-CA3 and subiculum, and ending at the border of the stratum radiatum (Fig. 4A,C). These looked similar to the interlaminar astrocytes of the neocortex. In surgical specimens, the long processes displayed small or no varicosities; in several autopsy specimens, the long processes displayed many large varicosities (Fig. 4B).

The hippocampus has an "inverted" position of pyramidal neurons in comparison with the neocortex-layer I of the neocortex corresponds to the stratum radiatum near the stratum lacunosummoleculare, and layer VI to the pyramidal layer near the stratum oriens. Thus, the long astrocyte processes covering the pyramidal layer corresponded to astrocyte processes going from subcortical white matter into layers V and VI. The stratum oriens is made up of axons of the pyramidal neurons and along with the alveus makes up the "white matter" of the hippocampus. The other hippocampal area where CD44+ astrocytes were found consistently is the molecular layer of dentate gyrus, where long processes of astrocytes in the subgranular layer cover the whole depth of the molecular layer (Fig. $4 D, E$ ). CD44+ astrocytes with long processes were also observed in the stratum radiatum (Fig. $4 F$ ) and in the hilus of the dentate gyrus (data not shown). Therefore, analysis of the hippocampus showed that CD44+ astrocytes with long processes are also typical for an archicortex population of astrocytes. The varicosities along the long processes in both cortex and hippocampus have been considered a typical feature of the long-process astrocytes (Akiyama et al., 1993; Oberheim et al., 2009). It is difficult to determine whether these varicosities are anatomic structures in living tissue or artifacts. We have left surgical temporal lobe material at room temperature for $15 \mathrm{~h}$ before paraformaldehyde fixation and found many more varicosities in long-process $\mathrm{CD} 44+$ astrocytes than in the same samples fixed immediately after surgery (A. Sosunov, unpublished observations). Thus, energy failure, either premortem or postmortem, may contribute significantly to the generation of varicosities, perhaps by an inhibition of cytoplasmic transport in astrocyte processes.

CD44+ astrocytes with long processes are phenotypically distinct from the protoplasmic astrocytes in gray matter and similar to fibrous astrocytes in white matter

We characterized the CD44+ astrocytes with long processes by their immunohistochemical profiles and compared them to protoplasmic and fibrous astrocytes. CD44+ astrocytes immunostained more weakly for EAAT2, EAAT1, and GS than did protoplasmic astrocytes (Figs. 2B,E, 5A-E). We quantified the optical density of GS immunolabeling and found that CD44+ astrocytes displayed significantly lower levels in comparison with neighboring CD44- protoplasmic astrocytes $(57.23 \pm 6.44$ vs 
$106.35 \pm 5.85$ a.u., respectively; $p<$ 0.001). The low EAAT1 and EAAT2 levels in CD44+ astrocytes do not mean, however, that the entire volume occupied by a CD44+ long-process astrocyte, even near their bodies, is devoid of astrocyte glutamate transporters. An examination of CD44 and EAAT1 or EAAT2 colabeling in the close vicinity of long-process astrocytes shows that there are indeed $\mathrm{EAAT} 1+$ and $\mathrm{EAAT} 2+$ processes intercalated between the CD44+ processes (Fig. $5 C, F)$. These EAAT1 + and EAAT2+ processes likely originate from neighboring CD44-, EAAT1+/EAAT2+, protoplasmic astrocytes (also see Astrocyte domain localizations and microheterogeneity among astrocyte types, below).

CD44+ astrocytes also showed consistent immunostaining for secreted protein acidic rich in cysteine (SPARC; osteonectin, in their cell bodies and processes; Fig. $6 A, A 1, B)$ and $\alpha \mathrm{B}$-crystallin (in their cell bodies; Fig. $6 C, C 1, D$ ), as is typical for fibrous astrocytes in white matter (Fig. $6 E, F)$ but is absent in protoplasmic astrocytes. The majority of CD44+ astrocytes displayed high levels of GFAP and S100 $\beta$, although some cells revealed low levels of immunoreactivity for these proteins.

AQP4 immunostaining was of special interest because of the close spatial relationship of the long-process astrocytes to blood vessels. All CD44+ astrocytes with long processes displayed positive immunostaining for AQP4 throughout the cell body and processes (Fig. 6G,H). This cell body and process staining appeared to be at a much higher level than that of protoplasmic astrocytes, which displayed mostly immunostaining at end feet (Fig. $6 G, H)$, suggesting a more widespread distribution of AQP4 over the entire cell rather than concentrated at blood vessel contacts. The $(\mathrm{CD} 44+)$ fibrous astrocytes of white matter also showed immunostaining for AQP4 over cell bodies and processes (data not shown).

Given that fibrous astrocytes in white matter contact nodes of Ranvier and participate in ion regulation in this area (Lundgaard et al., 2013), we examined the relation between CD44+ processes and nodes. Using Caspr1 as a paranodal junction marker, we could not find any contact between nodes and CD44+ long processes (neither at their ends nor along processes). CD44+ processes were only observed at some distance from the nodes (Fig. $6 I, I 1)$. In contrast, small processes of protoplasmic astrocytes were consistently found near nodes (Fig. 6J,J1,J2). Probably in gray matter, protoplasmic astrocytes participate in ionic buffering of extracellular space around nodes.

\section{Other types of CD44+ astrocytes within the neocortex}

In addition to the CD44+ astrocytes with long processes that descended or ascended into the cortex and the CD44+ longprocess astrocytes connected to large blood vessels, we observed
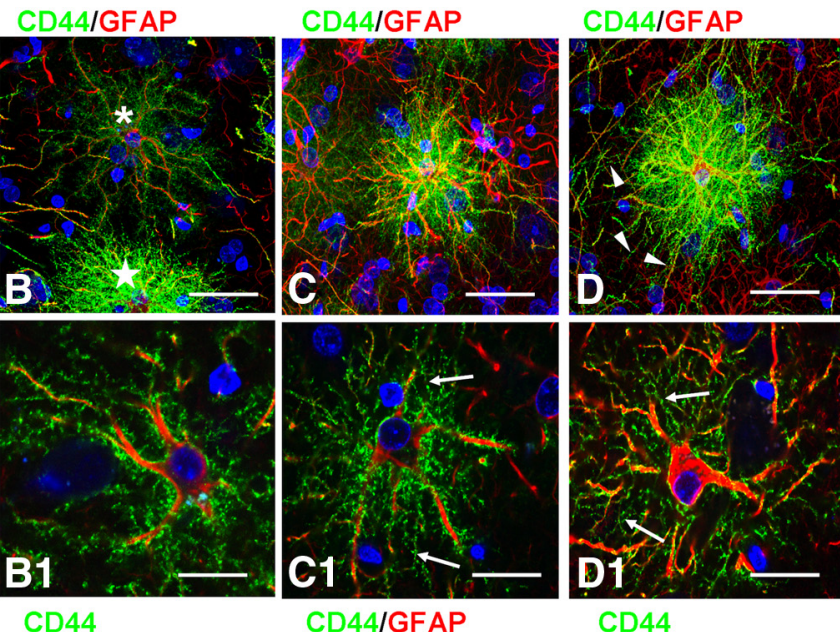

CD44/GFAP

CD44

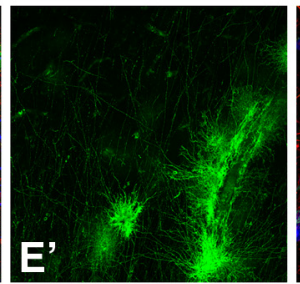

CD44

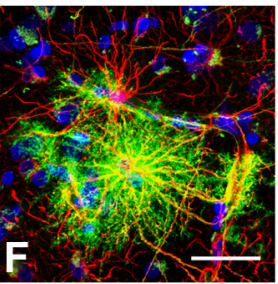

CD44/GFAP

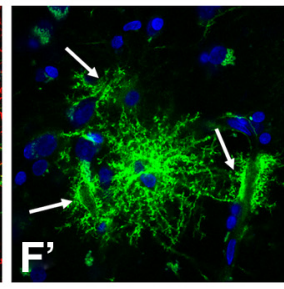

CD44
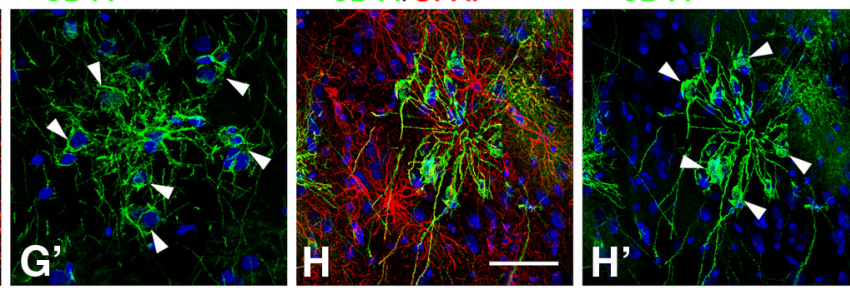

Figure 7. Variability in the morphologies of CD44+ cortical astrocytes without long processes. $A$, A bushy-like protoplasmic astrocyte. $\boldsymbol{B}$, Astrocyte (asterisk) with spongioform-like main processes with only few secondary and tertiary branches. Note astrocyte (star) with many processes nearby. C, D, CD44+ astrocytes with reduced numbers of miniature leaf-like

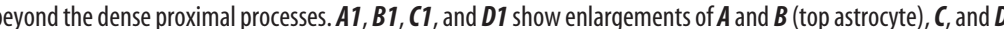
ayerIV Note the manylong CD44 astrocyte processes passing thoughe neuropil. $F$, CD44+ protoplasmic-like astrocyte with d feet on three separate blood vessels (arrows in $\boldsymbol{F}^{\prime}$ ). Note that end feet occupy large areas of blood vessel walls; single optical with Nissl staining used in the blue channel). Note that processes lack miniature processes. Confocal microscopy. Scale bars: $\boldsymbol{A}-\boldsymbol{C}$, D, $40 \mu \mathrm{m} ;$ A1, B1, C1, D1, $25 \mu \mathrm{m} ; \boldsymbol{E}, 145 \mu \mathrm{m} ; \boldsymbol{F}, 55 \mu \mathrm{m} ; \boldsymbol{G}, \boldsymbol{H}, 45 \mu \mathrm{m}$.

CD44+ astrocytes of variable morphologies within the cortex. The numbers of these cells varied considerably: in some specimens we found very few of them (Fig. 1A), while in others (55\% of studied cases, 32 of 58) they occupied all cortical layers (Fig. $1 B)$. Some of them ( $30 \%, 61$ of 200 cells) displayed CD44 immunostaining that homogeneously filled the entire volume occupied by the cell body and processes (Fig. 7A). Such bushy cells have the morphology of protoplasmic astrocytes and did not project long processes. Others ( $\sim 10 \%, 18$ of 200 cells) did not display a full bushy appearance, but rather showed a lower density of processes, many with small CD $44+$ protrusions, which corresponded to miniature leaflet-like astrocytic processes (Fig. $7 \mathrm{~B}, \mathrm{C})$. Most of the CD $44+$ astrocytes in this population $(\sim 60 \%$, 121 of 200 astrocytes) displayed a reduced density of miniature leaf-like processes on their main branches, and the appearance of many thin, short, curved processes originating from the perikarya and occupying the space between the proximal segments of main branches (Fig. $7 C, D$ ). These astrocytes often displayed a 

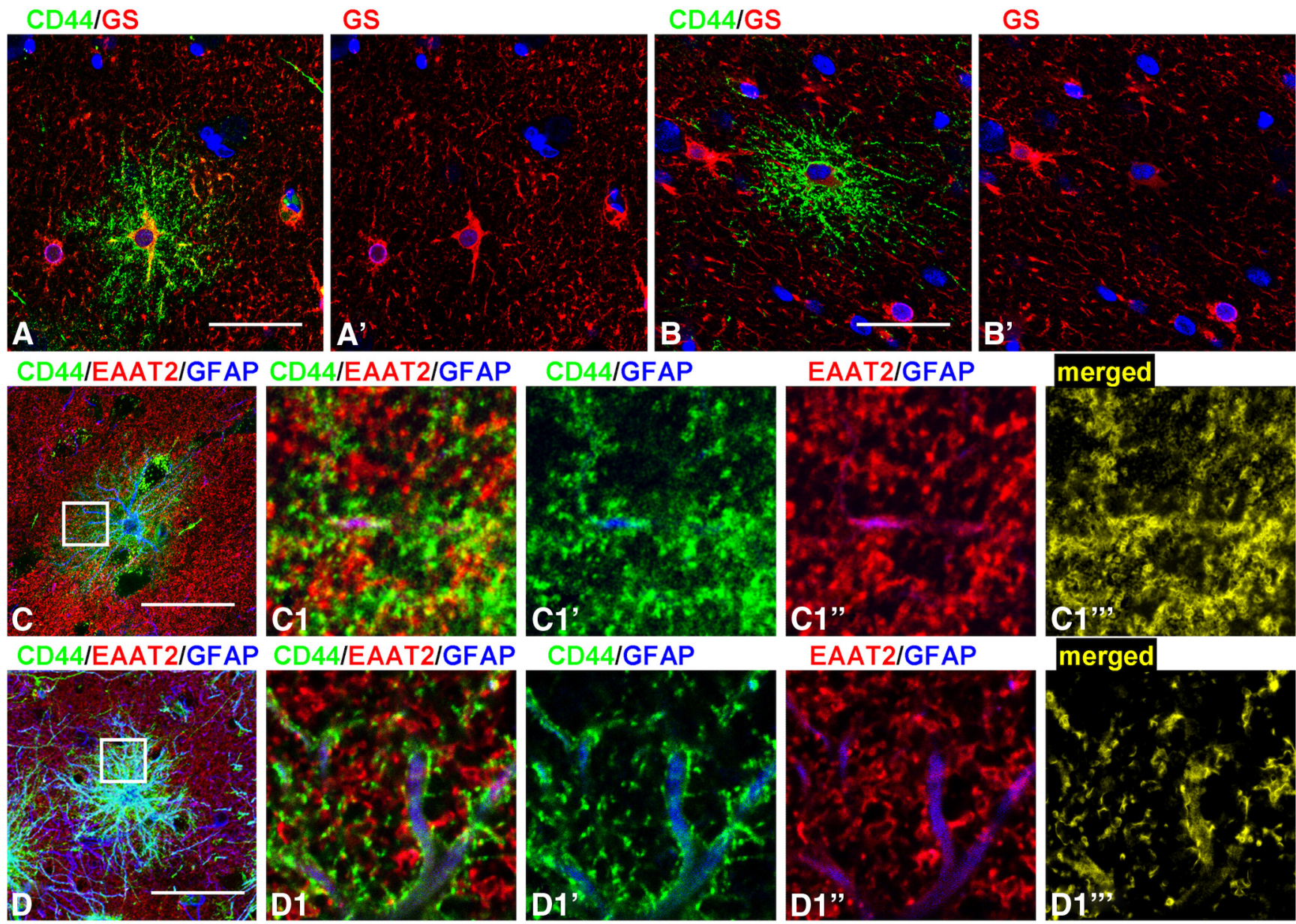

\section{CD44/GFAP}
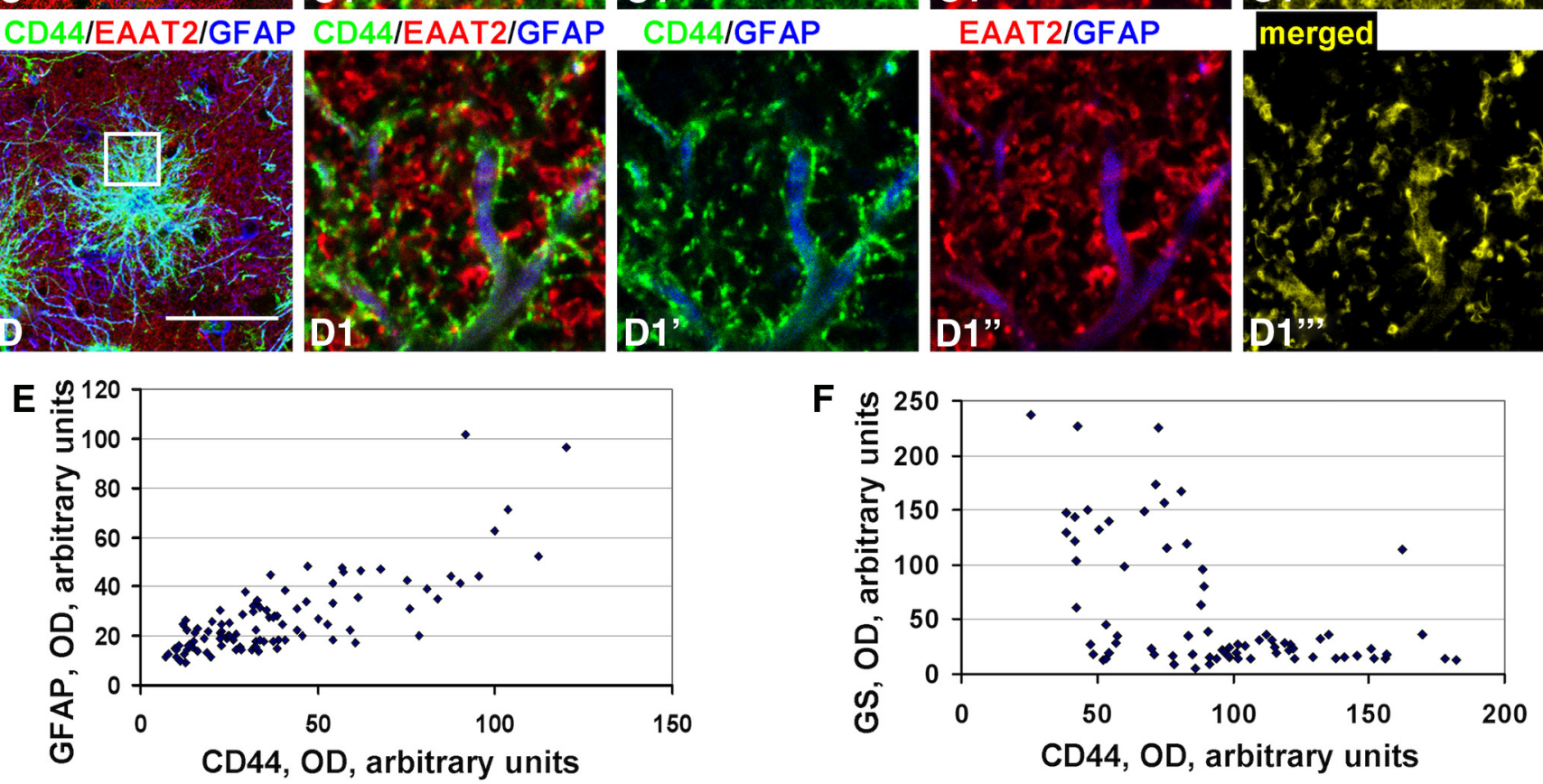

Figure 8. Variability in immunohistochemical properties of CD44+ cortical astrocytes without long processes. $A, B$, Different levels of $G S$ in $C D 44+$ astrocytes. C, D, Moderate $(C)$ and severe (D) decrease in EAAT2 immunolabeling in CD44+ astrocytes. (1, D1, Enlarged boxed areas in Cand D, respectively. C1"' and D1"', Areas of CD44 and EAAT2 colocalization (yellow): note the greater amount of overlap in $\mathbf{C 1}^{\prime \prime \prime}$ than in $\mathbf{D 1} \mathbf{1}^{\prime \prime \prime}$. Confocal microscopy. Scale bars: $\boldsymbol{A}, \boldsymbol{B}, 60 \mu \mathrm{m} ; \boldsymbol{C}, 100 \mu \mathrm{m} ; \boldsymbol{D}, 95 \mu \mathrm{m}$. $\boldsymbol{E}, 0$ ptical density reading of GFAP and CD44 in CD44+ astrocytes in mid-cortex. Note the significant direct correlation: $r=0.619, p<0.001 . F, 0$ ptical density reading of GS and CD44 in CD44+ astrocytes in mid-cortex. Note the significant inverse correlation: $r=0.456, p<0.001$.

few undulating, long processes that extended beyond the limits of the majority of the processes. It is worth noting that without confocal imaging many of the aforementioned CD $44+$ astrocytes looked bushy (Fig. 7C,D), but analysis of single optical sections revealed the reduction or absence of miniature leaf-like processes (Fig. 7C1,D1). Note also that these CD44+ astrocytes with a protoplasmic phenotype produced end feet that wrapped around a blood vessel (Fig. 7 E, F), in contrast to the CD44+ long-process astrocytes, whose end feet were small (Figs. 1E, 2B2,G). These astrocytes stained variably with anti-GS (Fig. $8 A, B$ ) and antiEAAT2 (Fig. 8C,D) antibodies.
One peculiar type of CD44+ astrocyte should be mentioned. These cells projected processes lacking secondary and miniature branches to neighboring neurons and embraced their perikarya with terminal branches (Fig. $7 G, H$ ). The number of these cells was not high $(<1 \%)$, but detailed examination of slices allowed us to find them in many of our samples. The majority of the CD44+ astrocytes in the mid-cortical layers also displayed high levels of GFAP immunostaining. An analysis of CD44 and GFAP optical densities for these cells showed a direct correlation between these markers $(r=0.619, p<0.001$; Fig. $8 E)$. Levels of EAAT2 and GS varied in these CD $44+$ astrocytes (Fig. $8 A-D, F$ ), 
and those with high levels of CD44 and GFAP were also AQP4 immunopositive (data not shown). We performed a regression analysis of GS and CD44 immunostaining based on optical density and determined an inverse correlation $(r=$ 0.456, $p<0.001$; Fig. $8 F$ ), indicating the significantly lower GS levels in these CD44+ astrocytes.

\section{Astrocyte domain localizations and microheterogeneity among astrocyte types}

Protoplasmic astrocytes, unlike fibrous astrocytes, spread their processes over a limited volume around their perikarya. This volume constitutes the astrocyte "domain." Only in a small, peripheral area do their processes intermingle with processes of neighboring astrocytes (Bushong et al., 2002, 2004). In contrast, the long processes of CD44+ astrocytes crossed through the domains of many protoplasmic astrocytes, often passing in close proximity to their perikarya (Fig. $9 A, B$; Oberheim et al., 2009, their Fig. 5A,F). As noted above, CD44+ astrocytes with long processes have lower or undetectable levels of EAAT2 immunostaining compared with protoplasmic astrocytes. Such a difference allowed us to determine the spatial relationships of the CD44+ longprocess astrocytes with their neighboring, CD44-, protoplasmic astrocytes. The EAAT2 + and EAAT1 + processes of protoplasmic astrocytes penetrated into the domains of neighboring CD44+ astrocytes (Figs. $5 F, 8 C, C 1, D, D 1$ ). This indicates that the breakdown of astrocyte domains is symmetrical-CD44+ astrocytes send their processes through many other astrocyte domains, and CD44protoplasmic astrocytes can send processes into a CD44+ astrocyte domain.

We also wanted to examine synaptic density in and around the volumes of the CD44+ long-process astrocytes. After immunostaining tissues with antibod-

ies to synaptophysin, we found many synaptophysin-positive puncta near cell bodies of CD44+ astrocytes (Fig. 9C-E). In fact, the density was not different from that within domains of EAAT2+/CD44- protoplasmic astrocytes. For CD44+ astrocytes with long processes, OD for synaptophysin puncta around CD44 + cell bodies ( $68.38 \pm 2.783$ a.u.) did not differ from that around nearby protoplasmic astrocytes $(69.093 \pm 2.832$ a.u.; $p=$ 0.448 ). For CD $44+$ astrocytes with short processes, we determined no correlation between levels of synaptophysin and CD44 immunoreactivity $(r=0.019, p<0.001$; Fig. $9 F)$. However, synaptophysin-positive puncta were located between the processes of CD44+ astrocytes, where the EAAT2+ processes of neighboring protoplasmic astrocytes resided. Such observations indicate that CD44+ astrocytes do not have isolated, exclusionary domains and that the volume between their processes, even

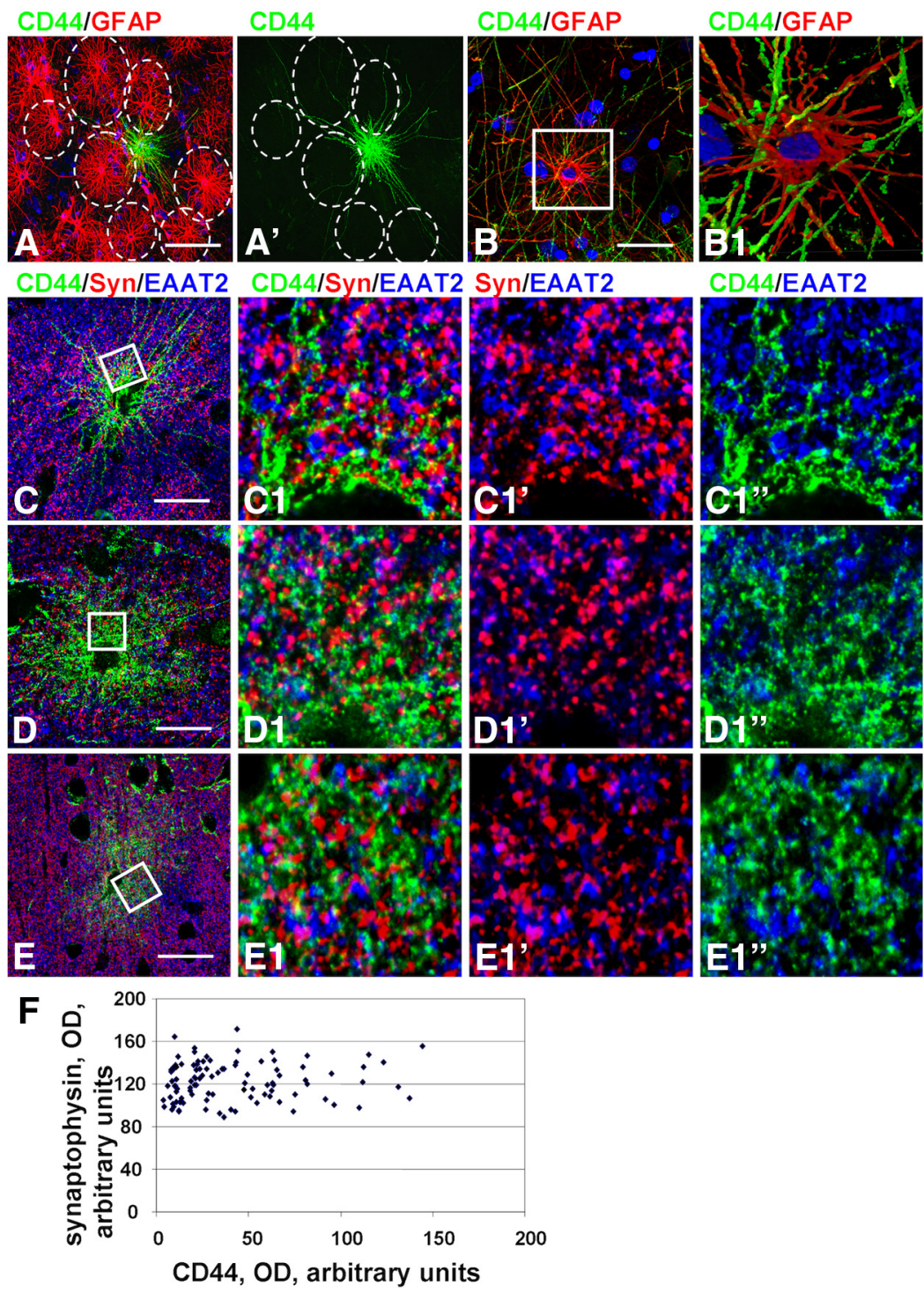

Figure 9. Protoplasmic astrocyte domain organization is violated by CD44+ astrocytes. $\boldsymbol{A}$, Long CD44+ processes pass through the domains (dotted lines) of neighboring CD44 - astrocytes. B, Many CD44+ long processes (green) pass through the processes of a CD44 - astrocyte. B1,2D image of 3D reconstruction of enlarged boxed area in $\boldsymbol{B}$. Note that CD44+ processes pass vicinity of $C D 44+$ astrocytes with long $(\boldsymbol{C})$ and short $(\boldsymbol{D}, \boldsymbol{E})$ processes. $\boldsymbol{C} \mathbf{1}, \mathbf{D} 1, \boldsymbol{E} 1$, Enlarged boxed areas in $\boldsymbol{C}-\boldsymbol{E}$, respectively; single optical slices. Note that there is less CD44 immunostaining in $\boldsymbol{C}$ than in $\boldsymbol{D}$ and $\boldsymbol{E}$. Confocal microscopy. Scale bars: $\boldsymbol{A}, 60 \mu \mathrm{m} ; \boldsymbol{B}, 45$ $\mu \mathrm{m} ; \boldsymbol{C}-\boldsymbol{E}, 55 \mu \mathrm{m} . \boldsymbol{F}, 0$ ptical density reading of synaptophysin puncta and CD44 in CD44 - and CD44 + astrocytes without long processes in mid-cortex. Note the absence of correlation, $r=0.0819, p<0.001$.

near the cell bodies, is occupied by processes of neighboring protoplasmic astrocytes and synapses.

A striking feature of the CD44+ astrocytes with long and short processes was the irregular and heterogeneous pattern of their localization in relation to protoplasmic astrocytes. Isolated $\mathrm{CD} 44+$ astrocytes might be found within areas of protoplasmic astrocytes (Figs. 5D,E, 8A, B). Blood vessels may be covered by both $\mathrm{CD} 44+$ and CD44- astrocytes (Fig. $10 A, A 1, B, D, E$ ). Note that end feet of the protoplasmic (short-process) astrocyte occupy much more vascular surface area than those of the longprocess astrocytes (Fig. 10D1,E1, compare short process to long process). We even found "doublets" of astrocytes that contained one CD44+ and one CD44- cell (Fig. 10C,C1). It is worth noting that all astrocytes with long processes when in doublets displayed similar levels of CD44 immunolabeling. 


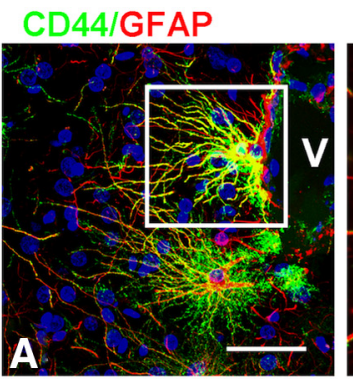

CD44/GS/GFAP

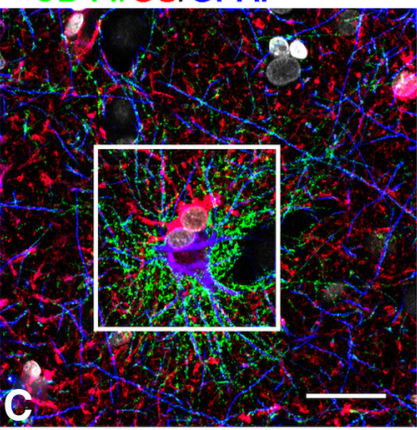

CD44/GFAP

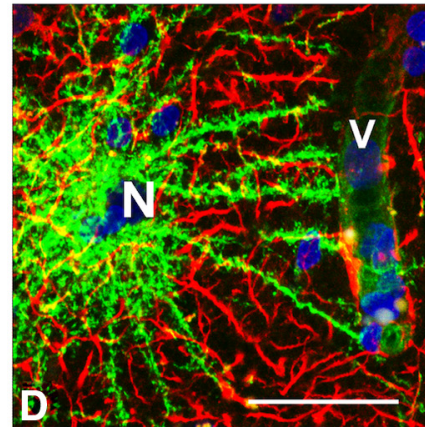

CD44/GFAP

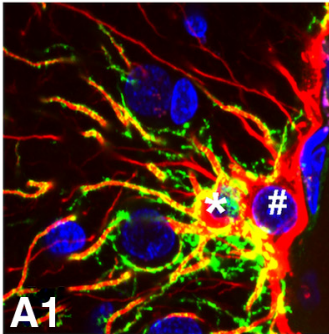

CD44/GS

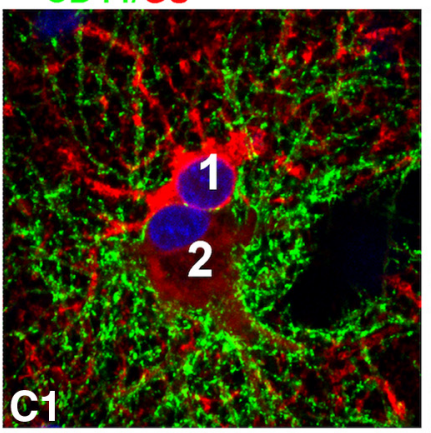

CD44

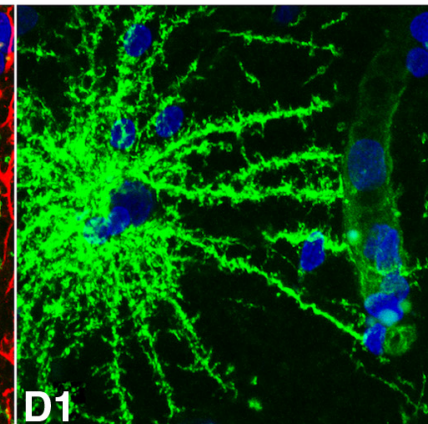

CD44

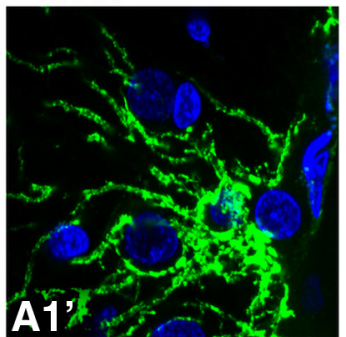

CD44

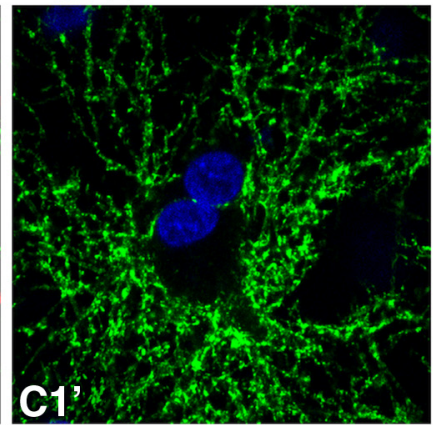

CD44/GFAP

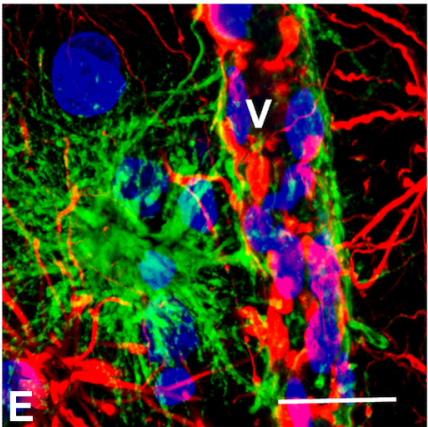

CD44/GFAP
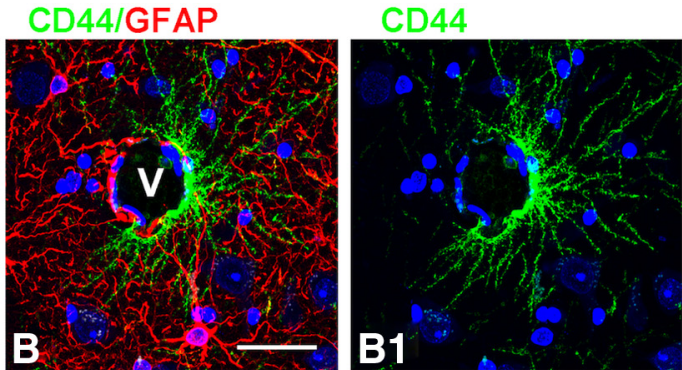

GFAP

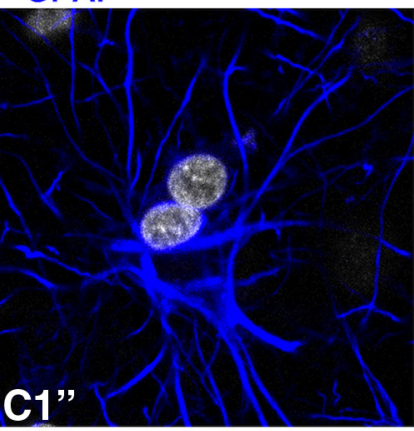

CD44

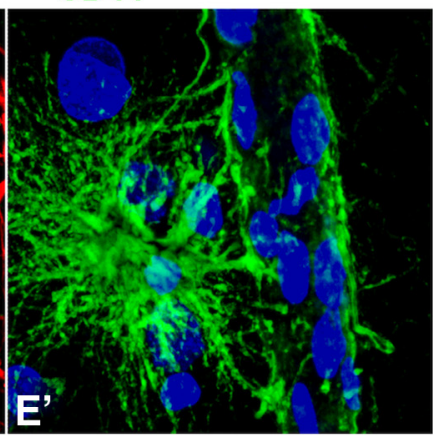

Figure 10. Microheterogeneity in astrocyte properties. $A$, Two neighboring perivascular astrocytes differ in CD44 levels. A1, Enlarged boxed area in $\boldsymbol{A}$. Note that the astrocyte marked with an asterisk is CD44+, whereas the other astrocyte (\#) is CD44- B, CD44+ astrocyte covers only half of the perimeter of a blood vessel (V). C, Two astrocytes (doublet) differ in CD44 levels and display different levels of GS and GFAP. C1, Enlarged boxed area in C. The numbers 1 and 2 indicate two astrocytes with different immunohistochemical phenotypes. $\boldsymbol{D}, \boldsymbol{E},(D 44+$ long-process astrocyte (D) and $(D 44+$ short-process astrocyte $(\boldsymbol{E})$ have different end feet. Note that end feet of the protoplasmic (short-process) astrocyte occupy much more vascular surface area. Note also in these images the different CD44+ and GFAP+ processes. Confocal microscopy. Scale bars: $A, 75 \mu \mathrm{m} ; \boldsymbol{B}, 90 \mu \mathrm{m} ; \boldsymbol{C}, 55 \mu \mathrm{m} ; \boldsymbol{D}, \boldsymbol{E}, 85 \mu \mathrm{m}$.

Neonatal brains contain CD44+ long-process astrocytes but not CD44+ cortical astrocytes

We examined fetal and neonatal telencephalons that did not display any gross or microscopic CNS pathology to determine when the CD44+ astrocytes appeared. As noted by Colombo et al. (2005) using a GFAP antibody, interlaminar processes are in place by several weeks of postnatal life and then increase in density for several months. In our samples, CD44+ astrocytes were not present in 19-21 week fetal brains (Fig. $11 A, B$ ), but CD44 immunostaining of pial-based astrocytes began to arise focally by 26 weeks of gestation (Fig. 11C). At term, we found that the long-process astrocytes with cell bodies at the pial surface and in lower cortex and superficial white matter were CD44+ (Fig. 11D,F). We also found that in these young brains, there were no CD44+ astrocytes with short processes (protoplasmic astrocytes) in the cortex itself (Fig. 11E). CD44+ fibrous astrocytes in deep white matter first appeared around midgestation and increased from that time (data not shown; Vogel et al., 1992). We also examined brains of young patients without seizures in the 2-7 year age range, and found no short process, CD44+ astrocytes in the midcortical layers (data not shown). To expand our observations, we also examined basal ganglia of full-term neonates and young children, and found long-process CD44+ astrocytes around large blood vessels (Fig. 11G). Thus, the long-process astrocytes are not specific for cortex, but are general residents of hemispheric gray matter and are associated with large vessels in gray matter areas.

\section{Discussion}

Pleomorphism and complexity of human astrocytes

The present study further strengthens the idea of astrocyte heterogeneity in the human brain, and the differences between human and rodent astrocytes (Colombo and Reisin, 2004; Oberheim et al., 2009). Immunostaining for CD44, a plasma membrane protein, outlines astrocyte morphology to a degree not possible with GFAP immunostaining. Typical locations and features of longprocess CD44+ astrocytes are shown in Fig. 12. Rodent astrocytes that are pial-based, contact large vessels, or reside in white matter are also CD44+ (Sosunov et al., 2013), but, to the best of our knowledge, CD44+ or CD44- long-process astrocytes have never been observed in rodent isocortex and hippocampus. The long-process interlaminar phenotype may in part be due to the large increase in brain size from birth to adulthood in humans, since astrocytes linked to the pia and blood vessels in the fetal brain would have to elongate as the brain grows. In addition, 
human astrocytes may have intrinsic properties that allow them to extend long processes, as shown after human astrocyte transplantation into mouse brain (Han et al., 2013).

\section{What are the origins of the CD44+ astrocytes in the neocortex?}

All of the specimens contained CD44+ astrocytes with long processes, principally those at the pial surface, those in white matter and deep cortical layers, and those associated with large vessels. The morphology and immunohistochemical profile of CD44+ astrocytes with long processes do not depend on the location of the cells (e.g., subpial area, near blood vessels, or in deep cortex) and are similar to those of fibrous astrocytes in white matter. These CD44+ long-process astrocytes are present in fetal and young brains, indicating they arise from a normal developmental program.

In addition to the long-process CD44+ astrocytes, we found CD44+ astrocytes within the cortex that varied considerably in number among different individuals. Some of these cells displayed the morphology of protoplasmic astrocytes and had no long processes. That we did not find these $\mathrm{CD} 44+$ astrocytes in the neonatal cortex and that their numbers are highly variable in adult brains suggest that this is an acquired phenotype. What might be the source of these intracortical CD $44+$ astrocytes? Do they arise from normal, CD44-, protoplasmic astrocytes in response to some sort of pathology?

Notably, we did not find in these CD44+ astrocytes some of the proteins typical of reactive changes (e.g., vimentin, nestin, p-S6), and they did not show the hypertrophic shape typical of reactive astrocytes observed after any type of brain insult. Furthermore, none of these astrocytes was part of a glial scar. The high variability in the phenotypes (from minimal to significant deviation from protoplasmic) of these astrocytes favors a gradual change of character, rather than an acute reactive response. We suggest that the appearance of these intracortical CD44+ astrocytes represents a long-term, gradual change similar to those found in age-related astrogliosis, defined by GFAP immunostaining. The gradual accumulation of GFAP only in some cortical protoplasmic astrocytes that produce a mosaic distribution of GFAP + and GFAPcells is a well known age-dependent phenomenon (Middeldorp and Hol, 2011). Similar heterogeneity in CD44+ astrocytes in the isocortex occurs with age in rhesus macaques (Cargill et al., 2012). It is unlikely that this age-related increase is secondary to major pathology, since these animals did not show evidence of neurodegeneration. What causes this age-related heterogeneity is not known. These cortical human CD44+ astrocytes presumably arise from normal, CD44-, protoplasmic astrocytes, perhaps in response to some sort of subclinical pathology acquired during life, such as hypoxic episodes, inflammation or infection, minor head trauma, or hypertension.
Many of our specimens were obtained from middle-aged patients with epilepsy, so we cannot exclude the possibility that seizures might have accelerated and aggravated astrocyte changes and promoted higher levels of GFAP and CD44. Nevertheless, some of the temporal lobe specimens from epileptic patients contained few or no CD44+ astrocytes in the mid-cortex. Furthermore, six of the specimens were from nonepileptic patients, and these specimens also showed variable numbers of CD44+ astrocytes. Even if the transition from CD $44-$ to CD $44+$ is a gradual one, or one caused by subclinical pathology, it does produce important changes in the astrocyte phenotype. In particular, astrocytes change their morphology and lose miniature leaf-like processes that normally envelop synapses, they accumulate GFAP, and display low levels of glutamate transporters (EAAT1 and EAAT2) and GS. These changes are very similar to those observed in the murine model of Alexander disease (Sosunov et al., 2013), in which the transition occurs due to the activation of intracellular stress pathways. We cannot ignore the possibility that some of these mid-cortical CD44+ astrocytes could have been generated anew from precursors in the adult CNS, but we have no direct evidence to support that conclusion. 


\section{isocortex}

pia

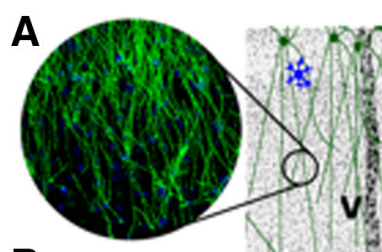

B
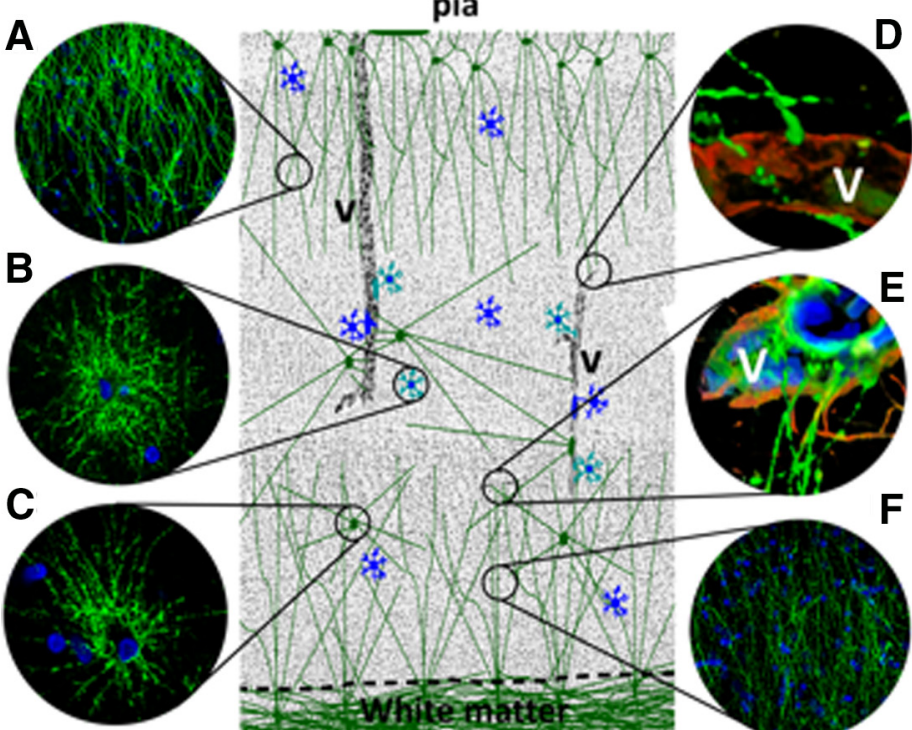

D

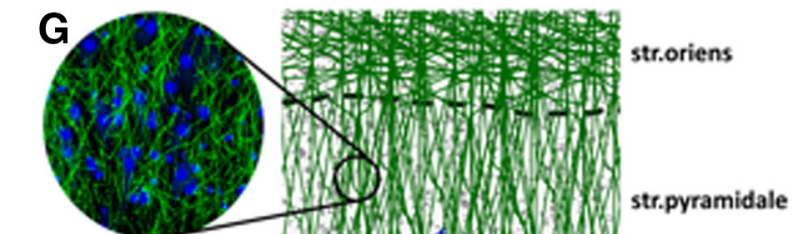

hippocampus
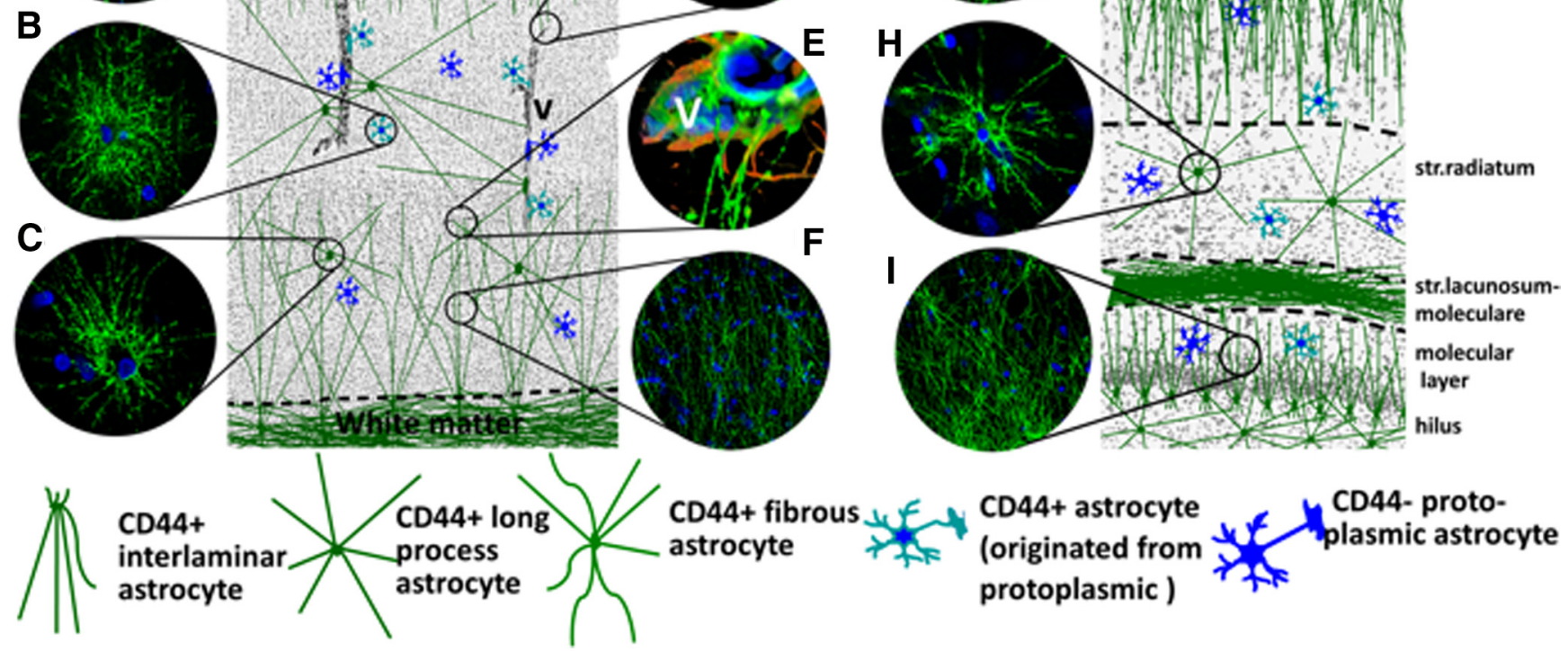

Figure 12. Schematic presentation of the locations of long-process CD44+ astrocytes in the isocortex and hippocampus. Note that in cortex both upper layers (mainly layers I and II) and deep layers (mainly layers VI and V) are covered with long processes of CD44+ astrocytes. The number of long-process CD44+ astrocytes in midcortex (layers III and IV) is lower. In hippocampus, long processes of CD44+ astrocytes occupy striatum pyramidale (but not striatum radiatum) and striatum moleculare of dentate gyrus. Protoplasmic CD44- astrocytes and short-process CD44+ astrocytes originating from CD44- protoplasmic astrocytes could be found in every cortical and hippocampal layer. Images in circles show typical features of CD44+ astrocytes. $A$, Descending long processes of subpial interlaminar astrocytes. $\boldsymbol{B}$, CD44+ astrocyte with short processes originated from CD44- protoplasmic astrocyte. C, CD44+ long-process astrocyte located in gray matter. $\boldsymbol{D}$, Process of interlaminar astrocyte ends with the end foot on a small blood vessel. $\boldsymbol{E}$, Blood vessel end feet made by CD44+ long-process astrocyte, and long processes of CD44+ fibrous astrocytes reside in white matter. $\boldsymbol{F}$, Long processes of fibrous astrocytes produce a dense net in deep cortical layers. $\boldsymbol{G}$, Long CD44+ processes of astrocyte reside in striatum oriens of hippocampus in striatum

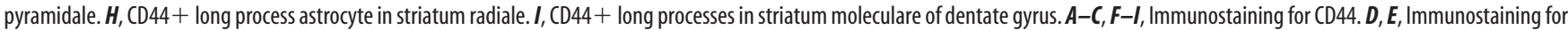
CD44 (green) and GFAP (red). V, Blood vessel.

What are the functions of CD44+ astrocytes with long processes?

CD44+ astrocytes with long processes contact many blood vessels and the pial surface. Thus, the CD44+ astrocytes cover much greater distances than protoplasmic astrocytes and are able to "connect" distant blood vessels to each other and deep blood vessels to the pial surface. The extent of vascular contact has not been appreciated in past studies, which found vessel contact but concluded that most of the long-process astrocytes did not contact vessels (Colombo et al., 1995; Reisin and Colombo, 2002b; Oberheim et al., 2009). Previous studies may not have appreciated the vessel attachments, since they visualized astrocytes with anti-GFAP antibodies.

Long-process astrocytes display AQP4 immunostaining over the entire cell body and processes in addition to end feet at vessels and the pial surface. AQP4 is the main astrocyte aquaporin responsible for water flux through the plasma membrane (Binder et al., 2012). Higher AQP4 levels along processes would allow efficient fluxes of water across the cell membrane. This would be particularly important in hyperosmolar states. Furthermore, long-process astrocytes may well constitute fast conduits for CSF components (a "glymphatic flux"; Iliff et al., 2012) over a large volume of brain parenchyma.

We found that half of the pial-based long-process astrocytes are coupled to neighboring $\mathrm{CD} 44-$ protoplasmic astrocytes.
This coupling would allow the protoplasmic astrocytes to link into a long-process distribution of water and ions. The longprocess astrocytes might significantly increase both the speed of communication and the volume of the astrocyte syncytium. The high speed of astrocytic calcium waves documented recently in human cortex (Oberheim et al., 2009) may be one such example.

Astrocytes are integral parts of neuron-vascular units and participate in the regulation of vascular tone (Gordon et al., 2007; Petzold and Murthy, 2011). Thus, astrocytes linked to multiple vessels widely apart could not only regulate vasotone locally but could also synchronize flow in distant vessels.

In contrast to fibrous astrocytes in white matter, CD44+ astrocytes in gray matter do not contact nodes. Buffering of the extracellular milieu near nodes is performed by protoplasmic astrocytes. Thus, blood vessels appear to be the major targets of the long processes of CD44+ astrocytes.

\section{CD44+ astrocytes do not respect the borders of} other astrocytes

Protoplasmic astrocytes generate their own domains, from which neighboring astrocytes are largely excluded (Bushong et al., 2002; Oberheim et al., 2009). CD44+ astrocytes with long processes represent an exception to this "rule of exclusion," since their long processes travel though the domains of many other astrocytes (Oberheim et al., 2009). Conversely, CD44- protoplasmic astro- 
cytes send their processes toward and settle close to the cell bodies of CD44+ astrocytes. Thus, protoplasmic astrocytes are able to extend their processes into the domain of an adjacent astrocyte when that astrocyte attenuates the "repulsive barrier" of its own domain. The extension of protoplasmic processes into a longprocess astrocyte domain would have the effect of bringing the fine processes of protoplasmic astrocytes near synapses in the regions of the long-process astrocytes, which do not extend such fine processes, and are low in GS and glutamate transporters. Thus, cortical synapses in the vicinity of long-process astrocytes still have "coverage" from protoplasmic astrocytes.

In summary, we infer that there are two general populations of CD44+ astrocytes in the human isocortex and hippocampus. The first is composed of the long-process astrocytes, which appear during normal development, and were present in every specimen we examined, regardless of age or other pathology. These cells generally show lower levels of GS, EAAT1, and EAAT2; variable but generally higher levels of GFAP and S-100 $\beta$; and higher levels of SPARC and $\alpha \mathrm{B}$-crystallin than protoplasmic astrocytes. The second is a much more heterogeneous population of cortical astrocytes, composed of cells that can appear protoplasmic in shape but with reduced numbers of miniature processes and rarely long processes that are devoid of small branches. This second population is variably immunopositive for GS, EAAT1, EAAT2, and GFAP. While the first population of CD44+ astrocytes is present throughout life, the second is likely to represent an acquired phenotype.

\section{References}

Akiyama H, Tooyama I, Kawamata T, Ikeda K, McGeer PL (1993) Morphological diversities of CD44 positive astrocytes in the cerebral cortex of normal subjects and patients with Alzheimer's disease. Brain Res 632: 249-259. CrossRef Medline

Andriezen WL (1893) The neuroglia elements in the human brain. Br Med J 29:227-230.

Arai Y, Takashima S, Becker LE (2000) CD44 expression in tuberous sclerosis. Pathobiology 68:87-92. CrossRef Medline

Bignami A, Dahl D (1986) Brain-specific hyaluronate-binding protein. A product of white matter astrocytes? J Neurocytol 15:671-679. Medline

Binder DK, Nagelhus EA, Ottersen OP (2012) Aquaporin-4 and epilepsy. Glia 60:1203-1214. CrossRef Medline

Botez G, Probst A, Ipsen S, Tolnay M (1999) Astrocytes expressing hyperphosphorylated tau protein without glial fibrillary tangles in argyrophilic grain disease. Acta Neuropathol 98:251-256. CrossRef Medline

Bushong EA, Martone ME, Jones YZ, Ellisman MH (2002) Protoplasmic astrocytes in CA1 stratum radiatum occupy separate anatomical domains. J Neurosci 22:183-192. Medline

Bushong EA, Martone ME, Ellisman MH (2004) Maturation of astrocyte morphology and the establishment of astrocyte domains during postnatal hippocampal development. Int J Dev Neurosci 22:73-86. CrossRef Medline

Cajal SRy (1984) Neuroglia. In: The neuron and the glial cell (de la Torre J, Gibson WC, eds.), pp. 263-290. Translation of Textura del Sistema Nervioso del Hombre y de los ertebrados (1937). Springfield, IL: C.C. Thomas.

Cargill R, Kohama SG, Struve J, Su W, Banine F, Witkowski E, Back SA, Sherman LS (2012) Astrocytes in aged nonhuman primate brain gray matter synthesize excess hyaluronan. Neurobiol Aging 33:830.e13-e24. CrossRef Medline
Colombo JA, Reisin HD (2004) Interlaminar astroglia of the cerebral cortex: a marker of the primate brain. Brain Res 1006:126-131. CrossRef Medline

Colombo JA, Yáñez Y, Puissant V, Lipina S (1995) Long, interlaminar astroglial cell processes in the cortex of adult monkeys. J Neurosci Res 40:551-556. CrossRef Medline

Colombo JA, Gayol S, Yañez A, Marco P (1997) Immunocytochemical and electron microscope observations on astroglial interlaminar processes in the primate neocortex. J Neurosci Res 48:352-357. CrossRef Medline

Colombo JA, Reisin HD, Jones M, Bentham C (2005) Development of interlaminar astroglial processes in the cerebral cortex of control and Down's syndrome human cases. Exp Neurol 193:207-217. CrossRef Medline

da Cruz LA, Cruz TF, Moscarello MA (1993) A glycoprotein expressed by human fibrous astrocytes is a hyaluronate-binding protein and a member of the CD44 family. Cell Adhes Commun 1:9-20. CrossRef Medline

Girgrah N, Letarte M, Becker LE, Cruz TF, Theriault E, Moscarello MA (1991) Localization of the CD44 glycoprotein to fibrous astrocytes in normal white matter and to reactive astrocytes in active lesions in multiple sclerosis. J Neuropathol Exp Neurol 50:779-792. CrossRef Medline

Gordon GR, Mulligan SJ, MacVicar BA (2007) Astrocyte control of the cerebrovasculature. Glia 55:1214-1221. CrossRef Medline

Han X, Chen M, Wang F, Windrem M, Wang S, Shanz S, Xu Q, Oberheim NA, Bekar L, Betstadt S, Silva AJ, Takano T, Goldman SA, Nedergaard M (2013) Forebrain engraftment by human glial progenitor cells enhances synaptic plasticity and learning in adult mice. Cell Stem Cell 12:342-353. CrossRef Medline

Iliff JJ, Wang M, Liao Y, Plogg BA, Peng W, Gundersen GA, Benveniste H, Vates GE, Deane R, Goldman SA, Nagelhus EA, Nedergaard M (2012) A paravascular pathway facilitates CSF flow through the brain parenchyma and the clearance of interstitial solutes, including amyloid $\beta$. Sci Trans Med 4:147ra111. CrossRef Medline

Kaaijk P, Pals ST, Morsink F, Bosch DA, Troost D (1997) Differential expression of CD44 splice variants in the normal human central nervous system. J Neuroimmunol 73:70-76. CrossRef Medline

Lundgaard I, Osório MJ, Kress B, Sanggaard S, Nedergaard M (2013) White matter astrocytes in health and disease. Neuroscience. Advance online publication. Retrieved January 6, 2014. doi:10.1016/j.neuroscience. 2013.10.050. CrossRef Medline

Middeldorp J, Hol EM (2011) GFAP in health and disease. Prog Neurobiol 93:421-443. CrossRef Medline

Oberheim NA, Takano T, Han X, He W, Lin JH, Wang F, Xu Q, Wyatt JD, Pilcher W, Ojemann JG, Ransom BR, Goldman SA, Nedergaard M (2009) Uniquely hominid features of adult human astrocytes. J Neurosci 29:3276-3287. CrossRef Medline

Petzold GC, Murthy VN (2011) Role of astrocytes in neurovascular coupling. Neuron 71:782-797. CrossRef Medline

Reisin HD, Colombo JA (2002a) Astroglial interlaminar processes in human cerebral cortex: variations in cytoskeletal profiles. Brain Res 937:5157. CrossRef Medline

Reisin HD, Colombo JA (2002b) Considerations on the astroglial architecture and the columnar organization of the cerebral cortex. Cell Mol Neurobiol 22:633-644. CrossRef Medline

Retzius G (1893) Studien über ependym und neuroglia. Biol Unters 5:2-26

Sosunov AA, Guilfoyle E, Wu X, McKhann GM 2nd, Goldman JE (2013) Phenotypic conversions of "protoplasmic" to "reactive" astrocytes in Alexander disease. J Neurosci 33:7439-7450. CrossRef Medline

Vogel H, Butcher EC, Picker LJ (1992) H-CAM expression in the human nervous system: evidence for a role in diverse glial interactions. J Neurocytol 21:363-373 30. CrossRef Medline

Zeltner L, Schittenhelm J, Mittelbronn M, Roser F, Tatagiba M, Mawrin C, Kim YJ, Bornemann A (2007) The astrocytic response towards invasive meningiomas. Neuropathol Appl Neurobiol 33:163-168. CrossRef Medline 